\title{
HPV-mediated nuclear export of HP1 $y$ drives cervical tumorigenesis by downregulation of p53
}

\author{
Sang $\mathrm{Ah} \mathrm{Yi}^{1}$. Dong Hoon Lee ${ }^{2} \cdot$ Go Woon Kim ${ }^{2} \cdot$ Hyun-Wook Ryu ${ }^{2}$. Jong Woo Park ${ }^{1}$ Jaecheol Lee ${ }^{1}$ Jihoon Han ${ }^{1}$. \\ Jee Hun Park ${ }^{1} \cdot$ Hwamok Oh${ }^{1} \cdot$ Jieun Lee ${ }^{1}$ Junjeong $\mathrm{Choi}^{2} \cdot$ Hyun-Soo Kim ${ }^{3} \cdot$ Hyeok Gu Kang $\mathbb{1}^{4,5} \cdot$ Da-Hyun Kim $^{4,5}$. \\ Kyung-Hee $\mathrm{Chun}^{4,5} \cdot$ Jueng Soo You ${ }^{6} \cdot$ Jeung-Whan Han ${ }^{1}$. So Hee Kwon ${ }^{2}$
}

Received: 10 August 2019 / Revised: 19 February 2020 / Accepted: 20 February 2020 / Published online: 23 March 2020

(c) The Author(s), under exclusive licence to ADMC Associazione Differenziamento e Morte Cellulare 2020. This article is published with open access

\begin{abstract}
E6 oncoprotein derived from high-risk human papillomavirus (HPV) drives the development of cervical cancer through p53 degradation. Because cervical cancer therapies to inactivate HPV or E6 protein are not available, alternative strategies are required. Here, we show that HPV-mediated nuclear export of human heterochromatin protein $1 \gamma(\mathrm{HP} 1 \gamma)$ reduces the stability of p53 through UBE2L3-mediated p53 polyubiquitination during cervical cancer progression. In general, HP1 plays a key role in heterochromatin formation and transcription in the nucleus. However, our immunostaining data showed that the majority of HP1 $\gamma$ is localized in the cytoplasm in HPV-mediated cervical cancer. We found that HPV E6 protein drives unusual nuclear export of HP1 $\gamma$ through the interaction between the NES sequence of HP1 $\gamma$ and exportin-1. The mutation of the NES sequence in HP1 $\gamma$ led to nuclear retention of HP1 $\gamma$ and reduced cervical cancer cell growth and tumor generation. We further discovered that HP1 $\gamma$ directly suppresses the expression of UBE2L3 which drives E6-mediated proteasomal degradation of p53 in cervical cancer. Downregulation of UBE2L3 by overexpression of HP1 $\gamma$ suppressed UBE2L3dependent $\mathrm{p} 53$ degradation-promoting apoptosis of cervical cancer cells. Our findings propose a useful strategy to overcome p53 degradation in cervical cancer through the blockage of nuclear export of HP1 $\gamma$.
\end{abstract}

Edited by J.M. Harwick

Supplementary information The online version of this article (https:// doi.org/10.1038/s41418-020-0520-5) contains supplementary material, which is available to authorized users.

Jeung-Whan Han

jhhan551@skku.edu

$\triangle$ So Hee Kwon

soheekwon@yonsei.ac.kr

1 Epigenome Dynamics Control Research Center, School of Pharmacy, Sungkyunkwan University, Suwon 16419, Republic of Korea

2 College of Pharmacy, Yonsei Institute of Pharmaceutical Sciences, Yonsei University, Incheon 21983, Republic of Korea

3 Department of Pathology, Severance Hospital, Yonsei University College of Medicine, Seoul 03722, Republic of Korea

4 Department of Biochemistry and Molecular Biology, Yonsei University College of Medicine, Seoul 03722, Republic of Korea

5 Brain Korea 21 PLUS Project for Medical Science, Yonsei University College of Medicine, Seoul 03722, Republic of Korea

6 Department of Biochemistry, School of Medicine, Konkuk University, Seoul 05029, Republic of Korea

\section{Introduction}

Cervical carcinogenesis is induced by persistent high-risk human papillomavirus (HPV) infection, which is different from other cancers or malignant diseases [1]. Among the fifteen carcinogenic HPV subtypes, high-risk HPV subtypes, including HPV16 and HPV18, are associated with $\sim 70 \%$ of the cervical cancers $[2,3]$. Although the incidence and mortality rates of patients with cervical cancer are decreasing due to effective diagnosis and vaccines, these methods do not cure patients who already have cervical cancer [4]. Moreover, currently existing therapeutic options, such as surgery, radiotherapy, and chemotherapy, are limited in patients with advanced or recurrent cervical cancers $[5,6]$. Thus, a significant number of studies are struggling to investigate targeted therapeutics using pathogenic mechanisms.

Oncogenic transformations by high-risk HPVs are mediated by the viral E6 and E7 oncogenes, which are regularly co-expressed [7]. The E7 oncoprotein dysregulates the cell cycle by inactivating the retinoblastoma tumor suppressor gene [8]. The E6 protein blocks p53-mediated 
growth arrest and apoptosis by inducing p53 degradation [9], which is dependent on E3 ubiquitin ligase, E6AP, and E2 ubiquitin-conjugating enzyme UBE2L3 [10-12]. Thus, the restoration of the p53 function by blocking E6 and E6AP-mediated degradation of p53 could be an attractive strategy for treating cervical cancers. However, to date, there have been no agents that can block the E6-specific degradation of p53 in HPV-driven cervical cancers.

Heterochromatin protein 1 (HP1), a histone code reader, specifically recognizes and binds to methylated histone $\mathrm{H} 3$ lysine 9 (H3K9me) $[13,14]$. The mammalian HP1 family, which comprises three isoforms ( $\mathrm{HP} 1 \alpha, \beta$, and $\gamma$ ), plays essential roles in various cellular processes including the heterochromatin organization, centromere stability, telomere stability, DNA repair, and cellular senescence [1518]. HP1 possesses three characteristic structural domains: an amino-terminal chromodomain (CD), a flexible hinge region, and a carboxyl-terminal chromoshadow domain (CSD) [19]. The HP1 CD binds H3K9me [13, 20, 21], whereas the CSD can homo- and heterodimerize and bind proteins containing the consensus sequence PXVXL $[19,22]$. HP1 isoforms exhibit different subnuclear localization in interphase: $\mathrm{HP} 1 \alpha$ and HP1 $\beta$ are centromeric whereas HP1 $\gamma$ is in both euchromatic and heterochromatic regions $[15,23]$, implying that $\mathrm{HP} 1 \gamma$ has more diverse functions not yet discovered. Several studies have shown that HP1 contributes to the progression of several cancers [24-26], while there are also evidences demonstrating that expression of HP1 isoforms is decreased in diverse tumor tissues [17]. Adding to these controversial findings, an indepth understanding of the role of HP1 in cancer progression would provide an interesting target for therapy.

Here, we found that abnormal nuclear export of HP1 $\gamma$, which is mediated by exportin-1, is responsible for E6dependent p53 degradation. Inhibition of exportin-1 or expression of nuclear export signal (NES)-depleted HP1 $\gamma$ blocked the nuclear export of HP1 $\gamma$ and subsequently restored p53 signaling reducing the tumorigenic potential of cervical cancer cells. Genome-wide analysis identified UBE2L3, which plays a role in p53 degradation, as a target gene of HP1 $\gamma$. Overexpression of HP1 $\gamma$ suppressed UBE2L3 and restored p53 stability, further inducing apoptosis of cervical cancer cells. These observations prompted us to take a closer look at the role of HP1 $\gamma$ in the cervical cancer and provide a pathogenic rationale to treat cervical cancer.

\section{Materials and methods}

\section{DNA constructs and antibodies}

The DNA constructs used in this study were pcDNA-EGFPHP1 $\beta$, pcDNA-EGFP-HP1 $\gamma$, pcDNA-EGFP-HP1 $\gamma$ V32A,
pcDNA-EGFP-HP1 $\gamma$ I165E, pcDNA-EGFP-HP1 $\gamma$ W174A, pcDNA-EGFP-HP1 $\gamma$ 150A, 152A, and pNCMV 16E6no*. The mutant constructs for HP1 $\gamma$ were generated using sitedirected mutagenesis (pcDNA-EGFP-HP1 $\gamma$ V32A, pcDNAEGFP-HP1 $\gamma$ I165E, pcDNA-EGPF-HP1 $\gamma$ W174A, and pcDNA-EGFP-HP1 $\gamma$ 150A, 152A). Anti-HP1 $\alpha$ (Epitomics, 5346-1), anti-HP1 $\beta$ (Millipore, MAB3448), anti-HP1 $\gamma$ (Millipore, 05-690), anti-p53 (Santa Cruz Biotechnology, SC-126), anti-UBE2L3 (abcam, ab108936), anti-ubiquitin (Santa Cruz Biotechnology, SC-9133), anti-Exportin-1 (Santa Cruz Biotechnology, SC-74454), anti-Bax (Santa Cruz Biotechnology, SC-20067), anti-Noxa (Santa Cruz Biotechnology, SC-56169), anti-Puma (Santa Cruz Biotechnology, SC-28226), anti-Caspase 3 (abcam, ab13585100), anti-Flag (Sigma, F-3165), anti-GFP (Santa Cruz Biotechnology, SC-9996), anti- $\alpha$-tubulin (Santa Cruz Biotechnology, SC-32293), anti-actin (Millipore, mab1501), and anti-Lamin A/C (Cell Signaling Technology, \#2032) antibodies were used in this study.

\section{Cell lines and treatment}

HeLa, SiHa, CaSki, and U2OS cells were purchased from the American Type Culture Collection (ATCC). The cells were cultured according to the instructions from ATCC and were maintained under a fully humidified atmosphere of $95 \%$ air and $5 \% \mathrm{CO}_{2}$ at $37^{\circ} \mathrm{C}$. To inhibit exportin-1, cells were treated with $50 \mathrm{nM}$ of leptomycin B (LMB) purchased from Sigma-Aldrich for $4 \mathrm{~h}$ or $100 \mathrm{nM}$ of KPT-330 purchased from Selleckchem for $24 \mathrm{~h}$.

\section{Doxycycline-inducible HP1Y expression}

The Tet-on 3G doxycycline-inducible expression system (Takara Bio USA, \#631354) was used to promote the expression of $H P l \gamma$ inserted under the TRE3G promoter in $\mathrm{SiHa}$ cells according to the manufacturer's protocol. After cloning HP1 $\gamma$ wild-type (WT) or AA mutant into the pLVX-TRE3G-IRES vector, we gathered lentiviral supernatants from 293T cells expressing both regulator vector (pLVX-EF1a-Tet3G) and response vector (pLVX-TRE3GIRES-HP1 $\gamma$ WT or pLVX-TRE3G-IRES-HP1 $\gamma$ AA). Then, $\mathrm{SiHa}$ cells were coinfected with the two viruses following G418 selection $(500 \mu \mathrm{g} / \mathrm{ml})$. After incubation with or without doxycycline $(1 \mu \mathrm{g} / \mathrm{ml})$ for additional $48 \mathrm{~h}$, the cells were harvested for analyses.

\section{Knockdown of gene expression}

Cells were transfected with siRNA targeting HP1 $\alpha$, HP $1 \beta$, HP1 $\gamma$, UBE2L3, or HPV16 E6 using Lipofectamine 2000 reagent (Life Technologies) according to the manufacturer's protocol. The sequences of siRNAs are as follow: 
HP1 $\alpha$ sense, 5'-GUUCCAGUCCUCUCUCAAAGC-3'

HP1 $\alpha$ antisense, 5'-GCUUUGAGAGAGGACUGGA AC-3';

HP1 $\beta$ sense, $5^{\prime}$-GACUCCAGUGGAGAGCUCAUG-3'

HP1 $\beta$ antisense, 5'-CAUGAGCUCUCCACUGGAG $\mathrm{UC}-3^{\prime}$;

HP1 $\gamma$ sense, 5'-AUUCUUCAGGCUCUGCCUC-3'

HP1 $\gamma$ antisense, 5'-GAGGCAGAGCCUGAAGAAU- $3^{\prime}$; UBE2L3 sense, $5^{\prime}$-UUUCUUUGUAAACUCUUCA-3'

UBE2L3 antisense, 5'-UGAAGAGUUUACAAAGAA A-3';

HPV16 E6 sense, 5'-CCACAGUUAUGCACAGAGC-3'

HPV16 E6 antisense, 5'-GCUCUGUGCAUAACUGU GG-3'.

\section{Immunohistochemistry}

Formalin-fixed and paraffin-embedded tissue sections were acquired from normal people and HPV-positive precancer, endocervical adenocarcinoma, and invasive squamous cell carcinoma patients. The 4- $\mu \mathrm{m}$ sections were deparaffinized in xylene and rehydrated through a graded alcohol series to distilled water. The antigen retrieval was performed by microwave irradiation, and the primary antibody against HP1 $\gamma$ (05-690, Millipore, diluted at 1:200) was then applied. The specific binding was detected with biotinylated anti-mouse immunoglobulin, followed by peroxidase-labeled streptavidin with 3,3'diaminobenzidine chromogen as substrate. Slides were counterstained with Harris hematoxylin. All protocols and procedures with human cervical tissues were approved by the Yonsei University Institutional Review Board (4-2017-0898).

\section{Immunoblotting and immunoprecipitation}

For immunoblotting, each sample was subjected to SDSpolyacrylamide gel electrophoresis. Proteins were transferred to polyvinylidene difluoride membranes using the semi-dry transfer (Bio-Rad). The membranes were incubated overnight with the indicated primary antibodies, followed by incubation with horseradish peroxidaseconjugated secondary antibodies for $1 \mathrm{~h}$ (Abcam). The signals were detected using chemiluminescence reagents (Intron). For immunoprecipitation, the cells were lysed with IP lysis buffer (HEPES $40 \mathrm{mM}$ (pH 7.4) containing $120 \mathrm{mM} \mathrm{NaCl}, 1 \mathrm{mM}$ EDTA, $50 \mathrm{mM} \mathrm{NaF}, 1.5 \mathrm{mM}$ $\mathrm{Na}_{3} \mathrm{VO}_{4}, 10 \mathrm{mM} \quad \beta$-glycerophosphate, 0.3\% CHAPS, and protease inhibitors). The lysates were centrifuged for $20 \mathrm{~min}$ at $13,000 \mathrm{rpm}$ at $4{ }^{\circ} \mathrm{C}$. The specific antibodies were incubated with the supernatants overnight at $4{ }^{\circ} \mathrm{C}$, followed by incubation with anti-rabbit Ig-IP beads (Trueblot) for $1 \mathrm{~h}$ at $4{ }^{\circ} \mathrm{C}$. The beads were spun-down for $1 \mathrm{~min}$ at $2000 \mathrm{rpm}$ and washed three times with IP wash buffer (IP lysis buffer without CHAPS). The proteins were eluted by boiling for $5 \mathrm{~min}$ in Laemmli buffer (Bio-Rad) and subjected to immunoblotting.

\section{Nuclear fractionation}

For the fractionation of cytoplasmic and nuclear extracts, cells were suspended in buffer A (10 mM HEPES containing $1.5 \mathrm{mM} \mathrm{MgCl}_{2}, 10 \mathrm{mM} \mathrm{KCl}, 1 \mathrm{mM}$ EDTA, $1 \mathrm{mM}$ DTT, $0.5 \mu \mathrm{g} / \mathrm{ml}$ leupeptin, $1 \mathrm{mM}$ PMSF, $1 \mu \mathrm{M}$ pepstatin A, and $0.05 \% \mathrm{NP}-40$ ), and cytoplasmic extracts were separated by centrifugation at $4{ }^{\circ} \mathrm{C}$ at $3000 \mathrm{rpm}$ for $10 \mathrm{~min}$. The remained pellet was resuspended in Buffer B $(20 \mathrm{mM}$ HEPES containing $1.5 \mathrm{mM} \mathrm{MgCl}, 420 \mathrm{mM} \mathrm{KCl}, 25 \%$ glycerol, $0.2 \mathrm{mM}$ EDTA, $1 \mathrm{mM}$ DTT, $0.5 \mu \mathrm{g} / \mathrm{ml}$ leupeptin, $1 \mathrm{mM}$ PMSF, and $1 \mu \mathrm{M}$ pepstatin A) and incubated on ice for $30 \mathrm{~min}$. Nuclear extracts were separated by centrifugation at $4{ }^{\circ} \mathrm{C}$ at $13,000 \mathrm{rpm}$ for $20 \mathrm{~min}$.

\section{Clonogenic assay}

For the stable cells, SiHa cells expressing doxycyclineinducible HP1 $\gamma$ WT or AA were seeded in $1 \times 10^{3}$ cells per well of a six-well plate and cultured with doxycycline $(0.5 \mu \mathrm{g} / \mathrm{ml})$ for $10-20$ days. For transiently expressing cells, HeLa or Siha cells were seeded in $2 \times 10^{3}$ cells per well of a six-well plate. Five days after, the cells were transfected with siRNA or DNA plasmids and the cells were grown 3-5 days more. Cells were fixed with glutaraldehyde $(6 \%)$ and stained with $0.5 \%$ crystal violet. The images of stained colonies were visualized using a digital scanner.

\section{Tumorigenesis with the xenograft mouse model}

All animal experiments were approved by the Institutional Review Board of the Yonsei University College of Medicine and were performed in specific pathogen-free facilities according to the university's guidelines for the Care and Use of Laboratory Animals (2018-0155). Female Balb/c nude mice (5-7 weeks old) were inoculated subcutaneously with $3 \times 10^{6}$ stable $\mathrm{SiHa}$ cells expressing pLVX-EF1a-Tet3G (control), pLVX-TRE3G-IRES-HP1 $\gamma$ WT, or pLVX-TRE3G-IRES-HP1 $\gamma$ AA into each flank under $100 \mu \mathrm{L}$ of saline/zoletil/rompun $(7: 1: 1)$ anesthesia. Mice were randomized into groups ( $n=8$ per group), and treatment was started 14 days after tumor implantation. Doxycycline (Duchefa, Netherlands) was administered to tumor-bearing animals orally, two times a week for 3 weeks at a dose of $50 \mathrm{mg} / \mathrm{kg}$. From palpable tumor formation until termination, tumor sizes were measured every 3-4 days using calipers, and tumor volume were 
calculated with the following formula: length $\times$ width $^{2} \times$ 0.5236 . Mice were sacrificed in a $7.5 \% \mathrm{CO}_{2}$ chamber, and tumors were harvested for immunohistochemistry and other analyses.

\section{Microarray}

RNA labeling and hybridization were performed by using the Agilent One-Color Microarray-Based Gene Expression Analysis protocol (Agilent Technology, V 6.5, 2010). Briefly, $200 \mathrm{ng}$ of total RNA from each sample was linearly amplified and labeled with Cy3-dCTP. The labeled cRNAs were purified by the RNAeasy Mini Kit (Qiagen). The concentration and specific activity of the

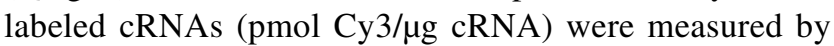
NanoDrop ND-1000 (NanoDrop). A total of $600 \mathrm{ng}$ of each labeled cRNA was fragmented by adding $5 \mu 10 \times$ blocking agent and $1 \mu \mathrm{l}$ of $25 \times$ fragmentation buffer, and then heated at $60^{\circ} \mathrm{C}$ for $30 \mathrm{~min}$. Finally, $25 \mu \mathrm{l}$ of $2 \times \mathrm{GE}$ hybridization buffer was added to dilute the labeled cRNA. Fifty microliters of hybridization solution was dispensed into the gasket slide and assembled to the SurePrint G3 Human Microarrays, $8 \times 60 \mathrm{~K}$ (Agilent Technologies, Inc). The slides were incubated for $17 \mathrm{~h}$ at $65^{\circ} \mathrm{C}$ in an Agilent hybridization oven and then washed at room temperature by using the Agilent One-Color Microarray-Based Gene Expression Analysis protocol (Agilent Technology, V 6.5, 2010). The hybridized array was immediately scanned with an Agilent Microarray Scanner (Agilent Technologies, Inc.). The cDNA microarray experiments (one-channel method) were repeated twice and the average of two gene expression values for each gene was used for further analysis. Statistical significance of the expression data was determined using LPE (Local Pooled Error) test and fold change in which the null hypothesis was that no difference exists among 2 groups. For statistical analysis, the LPE test was used for multiple group comparisons across all samples in the experiment by $\mathrm{MeV}$ (MultiExperiment Viewer) Software. A $p$ value of $<0.05$ was considered statistically significant and termed differentially expressed genes.

\section{Quantitative real-time PCR (qPCR)}

Total RNA was extracted using Easy-Blue reagent (iNtRON, Korea). Then $1 \mu \mathrm{g}$ of total RNA was reverse transcribed into cDNA using a Reverse Transcription kit (Promega, USA). Quantitative real-time PCR was performed using KAPATM SYBR FAST qPCR (KAPABIOSYSTEMS) with a CFX96TM or Chromo4TM real-time PCR detector (Bio-Rad). Relative levels of mRNA were normalized to the values of $G A P D H$ mRNA for each reaction. The qPCR primer sequences used are as follow:
GAPDH forward, 5'-GAGTCAACGGATTTGGTCGT-3'; GAPDH reverse, 5'-TTGATTTTGGAGGGATCTCG-3'; TP53 forward, 5'-GAGGGATGTTTGGGAGATGTAA GAAATG-3';

TP53 reverse, 5'-TTCACAGATATGGGCCTTGAAGT

TAGAGAA-3';

UBE2L3 forward, 5'-TTGACCCTT TGTAGGATTGG AATT-3';

UBE2L3 reverse, 5'-CGACCCCAGACTGGTGCTT-3';

Bax forward, 5'-TCTACTTTGCCAGCAAACTGG-3';

Bax reverse, 5'-TGTCCAGCCCATGATG GTTCT-3';

Noxa forward, 5'-AGAGCTGGAAGTCGAGTGT-3';

Noxa reverse, 5'-GCACCT TCACATTCCTCTC-3';

Puma forward, 5'-GACCTCAACGCACAGTA-3';

Puma reverse, 5'-CTAATTGGGCTCCATCT-3';

TP53AIP1 forward, 5'-TCTTCCTCTGAGGCGAGCT-3';

TP53AIP1 reverse, 5'-AGGTGTGTGTGTCTGAGCCC-3';

14-3-3 $\sigma$ forward, 5'-TTTCCTCT CCAGACTGACAA

ACTGTT-3';

14-3-3 $\sigma$ reverse, 5'-TAGAACTGAGCTGCAGCTGTA AA $-3^{\prime}$;

Gadd45 $\alpha$ forward, 5'-TGCGAGAACGACATCAACA $\mathrm{T}-3^{\prime}$;

Gadd45 $\alpha$ reverse, 5'-TCCCG GCAAAAACAAATAA G-3';

p21 forward, 5'-CACCGAGACACCACTGGAGG-3';

p21 reverse, 5'-GAGAAGATCAGCCGGCGTTT-3';

Tp53inpl forward, 5'-TGTTGCAGCTCTT GCTGCT CA-3';

Tp53inp1 reverse, 5'-GCTGATGAACAACCCAGCCA T-3';

HPV16 E6 forward, 5'-GAGTCAACGGATTTGGTC GT-3';

HPV16 E6 reverse, 5'-CATAAATCCCGAAAAGCAA AG-3'.

\section{Chromatin immunoprecipitation and qPCR (ChIP- qPCR)}

Total ChIP was performed as previously described [27]. In brief, a small portion of the cross-linked, sheared chromatin solution was reserved as the input DNA and the remainder was subjected to immunoprecipitation overnight at $4{ }^{\circ} \mathrm{C}$ using antibodies. After immunoprecipitation, the recovered chromatin fragments were subjected to qPCR using primer pairs specific for the target gene promoter. The primer sequences used for ChIP-qPCR are as follow:

Bax forward, 5'-TAATCCCAGCGCTTTGGAAG-3';

Bax reverse, 5'-TGCAGAGACCTGGATCTAGCA-3';

Noxa forward, 5'-CCTGGCCCCACCCCACCCCAC-3'; Noxa reverse, 5'-TCAGGGCTATTTTACGCTCTC- $3^{\prime}$; 14-3-3 $\sigma$ forward, $5^{\prime}$-TTTCCTCTCCAGACTGACAAA-3'; 14-3-3 $\sigma$ reverse, 5'-TAGAACTGAGCTGCAGCTGTA-3'; 
Gadd45 $\alpha$ forward, 5'-AGCGGAAGAGATCCCTGTG A-3';

Gadd $45 \alpha$ reverse, 5'-CGGGAGGCAGGCAGATG-3'.

\section{Flow cytometry}

Apoptosis of cervical cancer cells was assessed using Annexin V-PI double staining as previously described [28]. Cells were trypsinized and stained with $0.5 \mathrm{mg} / \mathrm{ml}$ Annexin $\mathrm{V}$ in binding buffer (10 $\mathrm{mM}$ HEPES free acid, $0.14 \mathrm{M} \mathrm{NaCl}$, and $2.5 \mathrm{mM} \mathrm{CaCl} 2)$ for $15 \mathrm{~min}$. Afterward, PI $(5 \mathrm{mg} / \mathrm{mL}$ final concentration) was added and incubated for another $15 \mathrm{~min}$, then applied to a flow cytometer for data collection.

\section{Survival analysis of cervical cancer patients}

The normalized level 3 TCGA RNA sequencing data of cervical cancer were retrieved from https://gdac.broa dinstitute.org. A total of 61 patients with available clinical information was analyzed for overall survival and diseasefree survival. Kaplan-Meier survival curves and log-rank statistics were employed to evaluate time to tumor recurrence and overall survival. Statistical significance was when $p<0.05$.

\section{Statistical analysis}

Statistical significance was analyzed using Student's $t$ test (two-tailed) and Pearson's correlation test. Statistical differences were determined based on a $P$ value $\left({ }^{*} p<0.05\right.$, $* * p<0.01$, and $* * * p<0.001)$.

\section{Results}

\section{Abnormal nuclear export of HP1 $\mathrm{Y}$ is induced by high-risk HPV E6 protein}

To gain insight into the putative role of HP1 on cancer progression, we examined the subcellular localization of three HP1 isoforms in diverse cancer samples by analyzing publicly available immunohistochemistry data of The Human Protein Atlas (HPA) project. HPA analysis showed cervical cancer-specific cytoplasmic localization of HP1 $\gamma$, whereas no distinctive cancer type-specific localization of HP1 $\alpha$ and HP1 $\beta$ was observed (Supplementary Fig. S1). Thus, we subjected cervical cancer tissue specimens to immunohistochemical analysis for the staining of HP1 $1 \gamma$. Unlike that observed in normal specimens, in which HP1 $\gamma$ was detected mainly in the nuclear region (Fig. 1a, upper), HP1 $\gamma$ was diffused from the nucleus to the cytoplasmic region in HPV-positive adenocarcinoma specimens (Fig. 1a, lower). However, HP1 $\gamma$ in HPV-positive cervical tissues from the precancer stage was detected only in the nucleus, whereas cytoplasmic localization of HP1 $\gamma$ in cervical tissues was observed in both adenocarcinoma and squamous cell carcinoma (Supplementary Fig. S2). TCGA survival analysis showed that the survival of cervical cancer patients was not significantly associated with the expression level of $C B X 3$ (a gene encoding HP1 $\gamma$ ) (Supplementary Fig. S3). These data indicate that subcellular localization of HP1 $\gamma$, rather than its expression, is closely involved in the progression of cervical cancer.

We next assessed whether the E6 oncoprotein of highrisk HPV is responsible for the nuclear export of HP1 $\gamma$. Nuclear fractionation analysis after transfection of HPV16 E6 showed an increase in the level of cytoplasmic HP1 $\gamma$ and a reduction in the level of nuclear HP1 $\gamma$, both in HPVnegative (C33A) and -positive ( $\mathrm{SiHa}$ ) cells (Fig. 1b, Supplementary Fig. S4A), implying immediate nuclear export of HP1 $\gamma$ by high-risk HPV infection. Furthermore, knockdown of E6 protein using siRNA targeting HPV16 E6 in SiHa cells (Supplementary Fig. S4B) blocked the nuclear export of HP1 $\gamma$ (Fig. 1c). Several studies have reported that E6 protein from HPV forces nuclear export of p53, which leads to degradation of $\mathrm{p} 53$ by cytoplasmic proteasomes [29-31]. We also observed that HPV E6 contributed to the reduction of p53 protein levels in both subcellular parts, cytoplasm and nucleus (Fig. 1b, c). While a majority of HP1 $\gamma$ research has focused on its chromatic role, our data provide insights into the cytoplasmic localization of HP1 $\gamma$, which occurs during HPV infection-mediated cancer progression.

\section{Exportin-1-mediated nuclear export of HP1Y is responsible for $\mathrm{p} 53$ downregulation in cervical cancer}

Earlier studies have reported that exportin-1 (encoded by $X P O 1)$ is involved in high-risk HPV-mediated elevation of the nuclear export system [29-32]. Moreover, the analysis of GEO profiles (GDS3233) showed a significant increase in the expression of XPO1 in cervical cancer cell lines and cervical cancer tissues, compared with normal cervix epithelium tissues (Fig. 1d). In parallel with this finding, the binding of HP1 $\gamma$ to exportin-1 was significantly increased after the overexpression of HPV16 E6 protein (Fig. 1e). This interaction was ablated by treatment with exportin-1 inhibitor, LMB (Fig. 2a), which subsequently induced the nuclear accumulation of HP1 $\gamma$ (Fig. 2b). Nuclear export of p53 was also inhibited by LMB treatment (Fig. 2b), which upregulated the p53 protein level and p53-mediated transcription of its target genes such as Bax, Noxa, 14-3-3 $\sigma$, and Gadd $45 \alpha$ (Fig. 2c-e). LMB-mediated increase in p53 target gene expression was disturbed by knockdown of p53 (Fig. 2e). Similar effects were produced by KPT-330, 
Fig. 1 Aberrant nuclear export of HP1 $\gamma$ is induced by highrisk HPV E6 protein. a Immunohistochemical staining of HP1 $\gamma$ protein in cervical tissues from normal specimens (upper) and HPV-positive cervical adenocarcinoma patients (lower). b Immunoblot analysis of cytoplasmic and nuclear extracts from $\mathrm{C} 33 \mathrm{~A}$ cells expressing Flag-HPV16 E6.

c Immunoblot analysis of cytoplasmic and nuclear extracts from $\mathrm{SiHa}$ cells expressing siRNA of HPV16 E6. d Boxand-Whisker plot of relative $X P O 1$ expression in cervical cancer cell lines $(n=9)$, normal tissues $(n=24)$, and cervical cancer tissues $(n=28)$

(GDS3233). e Immunoblot analysis of HP1 $\gamma$ immunoprecipitates (IP) and whole-cell lysates (WCL) from C33A cells expressing FlagHPV16 E6. a
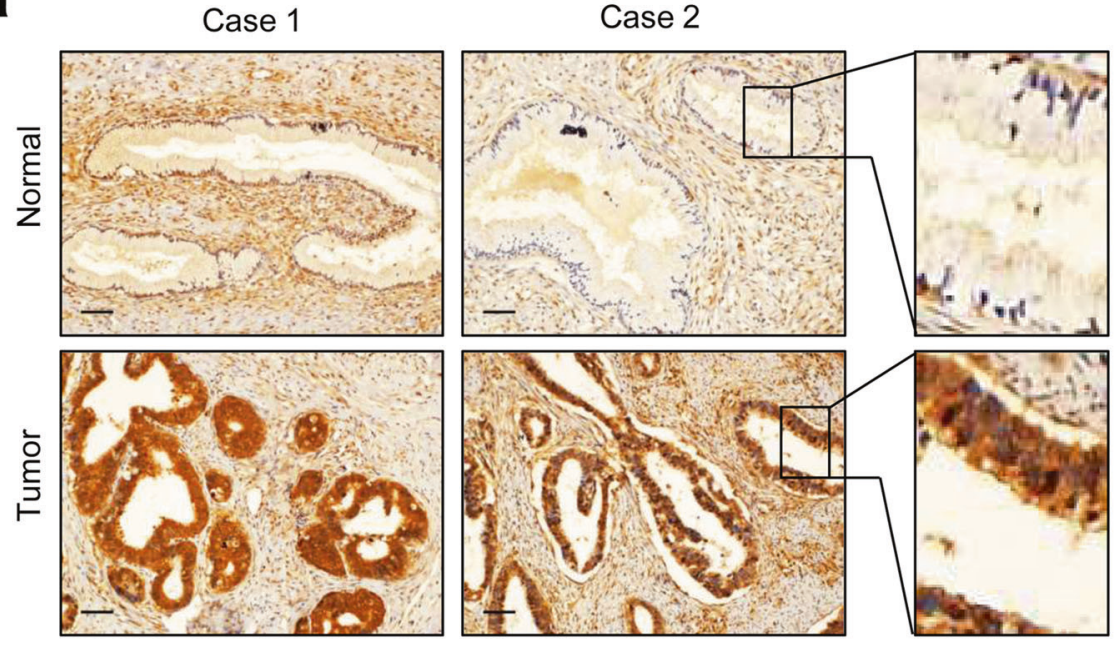

b

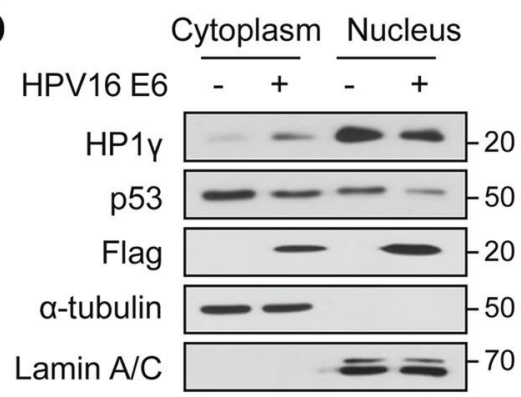

C
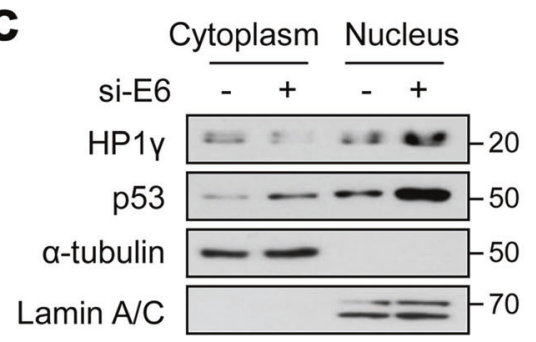

d

GDS3233

e

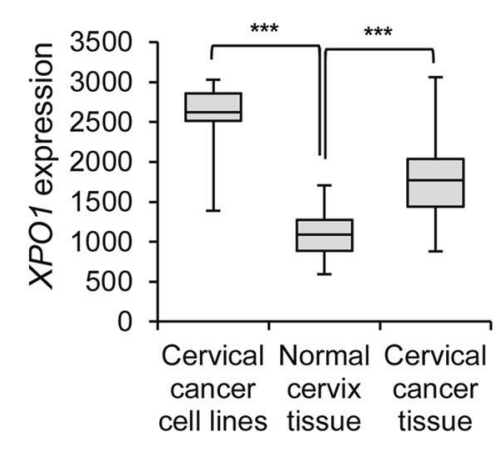

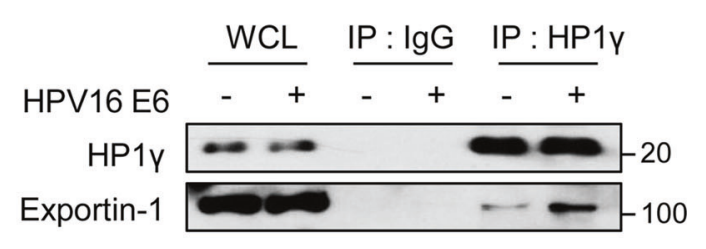

another exportin-1 inhibitor (Supplementary Fig. S4C, D). These results suggest that exportin-1 is responsible for the abnormal nuclear export of HP1 $\gamma$ as well as p53.

Given that exportin-1 usually interacts with the leucinerich site of its target protein, known as a NES sequence [33], we generated an NES mutant of HP1 $\gamma$ containing double alanine mutation (AA) at leucine residues 150 and 152 (Fig. 2f). The interaction between HP1 $\gamma$ AA mutant and exportin-1 was markedly reduced compared with WT HP1 $1 \gamma$ (Fig. 2g), accompanied by the nuclear accumulation of the HP1 $\gamma$ AA mutant as assessed by immunofluorescence (Fig. 2h) and cell fractionation (Fig. 2i). Intriguingly, expression of the HP1 $\gamma$ AA mutant led to increases in protein levels of p53 and apoptotic markers (Fig. 2j) as well as the transcription of p53 target genes (Fig. 2k), compared with those in WT HP1 $\gamma$-expressing cells. Thus, the interaction between exportin- 1 and the NES of HP1 $1 \gamma$ transports HP1 $\gamma$ from the nucleus to the cytoplasm, likely involved in the reduction of $\mathrm{p} 53$.

\section{Inhibiting nuclear export of HP1 $\gamma$ ameliorates tumorigenesis of cervical cancer}

To investigate whether nuclear export of HP1 $\gamma$ is involved in tumorigenesis of cervical cancer, we generated stable cell lines overexpressing HP1 $\gamma$ using doxycycline-inducible 
a

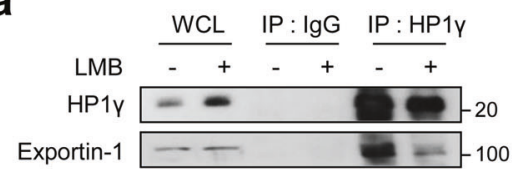

b

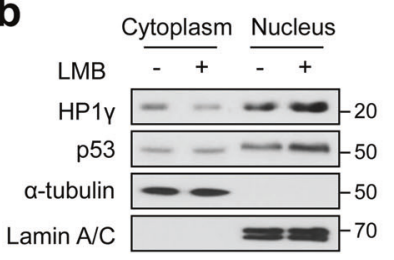

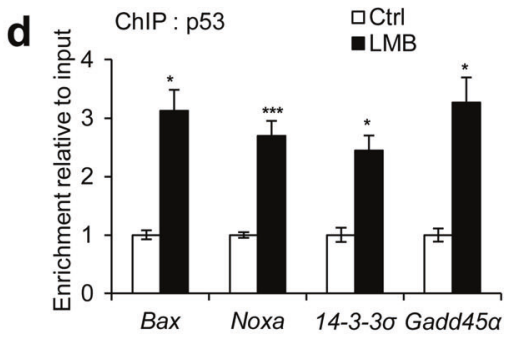

C

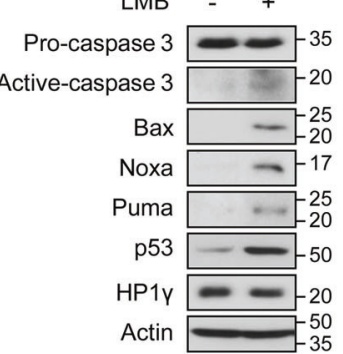

e

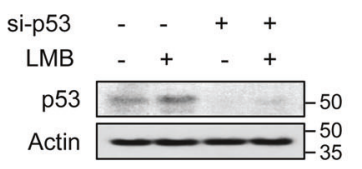

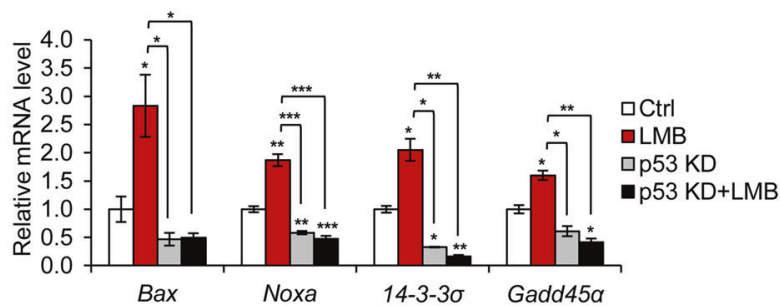

f

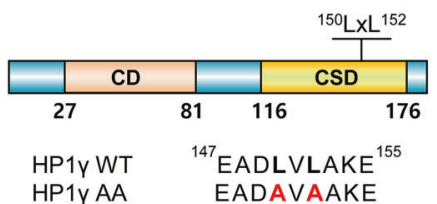

g

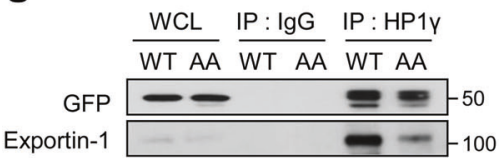

h

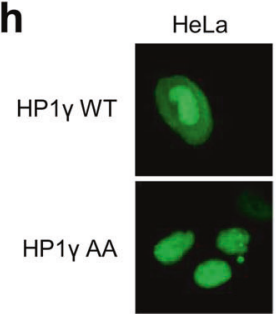

$\mathrm{SiHa}$

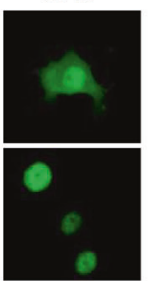

i

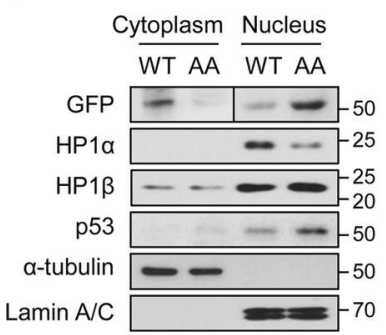

j

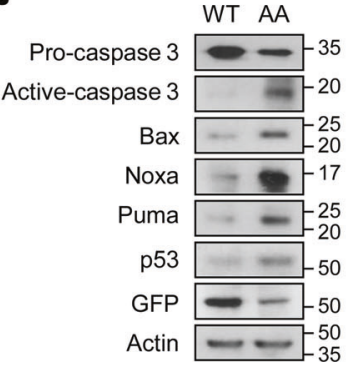

Fig. 2 Exportin-1-mediated nuclear export of HP1 $\gamma$ contributes to p53 degradation. a Immunoblot analysis of HP1 $\gamma$ immunoprecipitates (IP) and whole-cell lysates (WCL) from HeLa cells treated with or without LMB. b Immunoblot analysis of cytoplasmic and nuclear extracts from $\mathrm{SiHa}$ cells treated with or without LMB. c Immunoblot analysis of $\mathrm{SiHa}$ cells treated with or without LMB. d SiHa cells were treated with or without LMB, followed by ChIP-qPCR analysis with a p53 antibody in the promoter regions of p53 target genes. e Immunoblot analysis and the mRNA levels of p53 target genes in SiHa cells expressing siRNA of p53 in the presence or absence of LMB. f Representation of HP1 $\gamma$ protein and partial CD sequence containing nuclear export signal (NES) sequences (L150 and L152) which are

system (Fig. 3a). In line with the data from transiently HP1 $\gamma$-expressing cells (Fig. 2i), stable expression of HP1 $\gamma$ AA slightly decreased the protein level of HP1 $\alpha$ without alterations in the level of HP1 $\beta$ (Fig. 3a). Clonogenic assay data showed that overexpression of WT HP1 $\gamma$ markedly reduced colony formation of SiHa cells, which was further suppressed by long-term expression of HP1 $1 \gamma$ AA mutant (Fig. 3b). Next, we evaluated the in vivo tumorigenesis of HP1 $\gamma$-expressing SiHa cells in the xenograft mouse model. The tumorigenic capacity of SiHa cells was impeded by

k

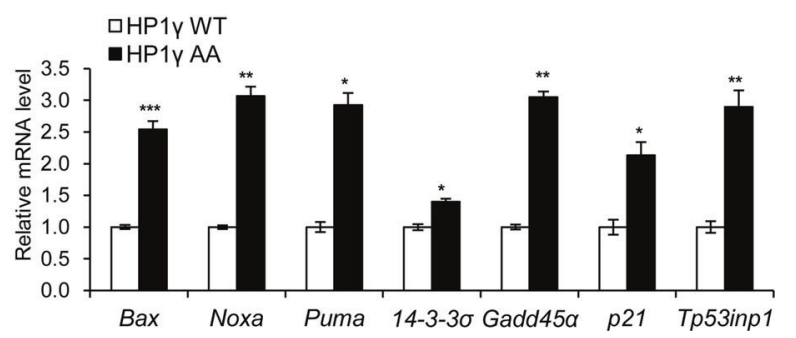

substituted with alanine in the mutant vector, HP1 $\gamma$ AA. g Immunoblot analysis of HP1 $\gamma$ immunoprecipitates (IP) and whole-cell lysates (WCL) from SiHa cells expressing GFP-HP1 $\gamma$ WT or AA mutant. h Immunofluorescence image of GFP-HP1 $\gamma$ WT or AA mutant expressed in $\mathrm{HeLa}$ (left) and $\mathrm{SiHa}$ (right) cells. i Immunoblot analysis of cytoplasmic and nuclear extracts from $\mathrm{SiHa}$ cells expressing GFPHP1 $\gamma$ WT or AA mutant. j Immunoblot analysis of SiHa cells expressing GFP-HP1 $\gamma$ WT or AA mutant. $\mathbf{k}$ The mRNA levels of p53 target genes in SiHa cells expressing GFP-HP1 $\gamma$ WT or AA mutant. Data are presented as the mean $\pm \operatorname{SEM}(n=3)$. $* P<0.05 ; * * P<0.01$; $* * * P<0.001$.

overexpression of HP1 $\gamma$, particularly in the HP1 $\gamma$ AA mutant group (Fig. 3c). Quantification of tumor volume and tumor weight showed a significant reduction in tumor growth in the HP1 $\gamma$ AA mutant group, and a lesser extent in WT HP1 $\gamma$ group (Fig. 3d, e). We then observed the expression pattern of HP1 $\gamma$ by staining the histological sections of tumor tissues from the xenograft mice. While WT HP1 $\gamma$ was diffused to the cytoplasm in tumor cells (Fig. 3f, middle), the HP1 $\gamma$ AA mutant was predominantly localized at the nucleus (Fig. 3f, right). These data imply the 

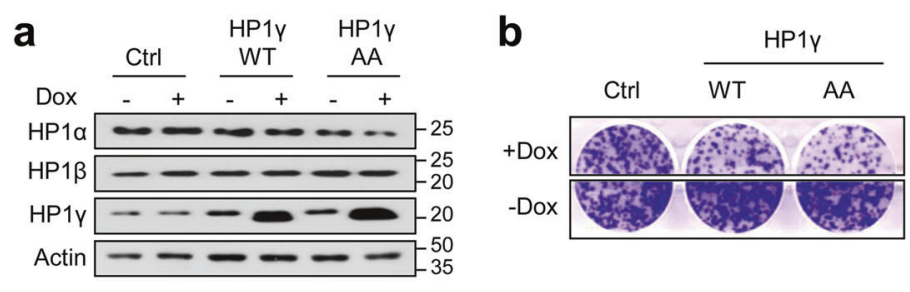

d

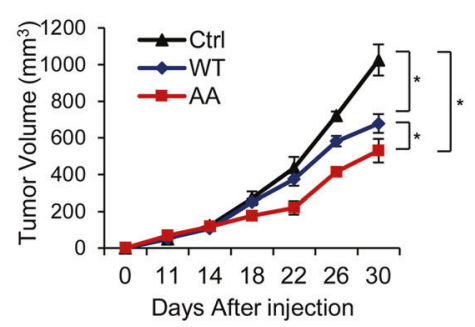

e

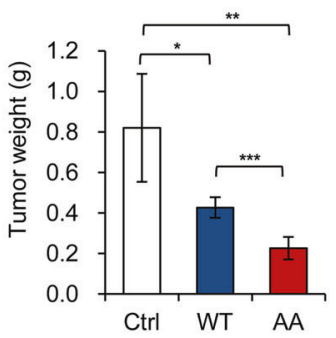

\section{f}
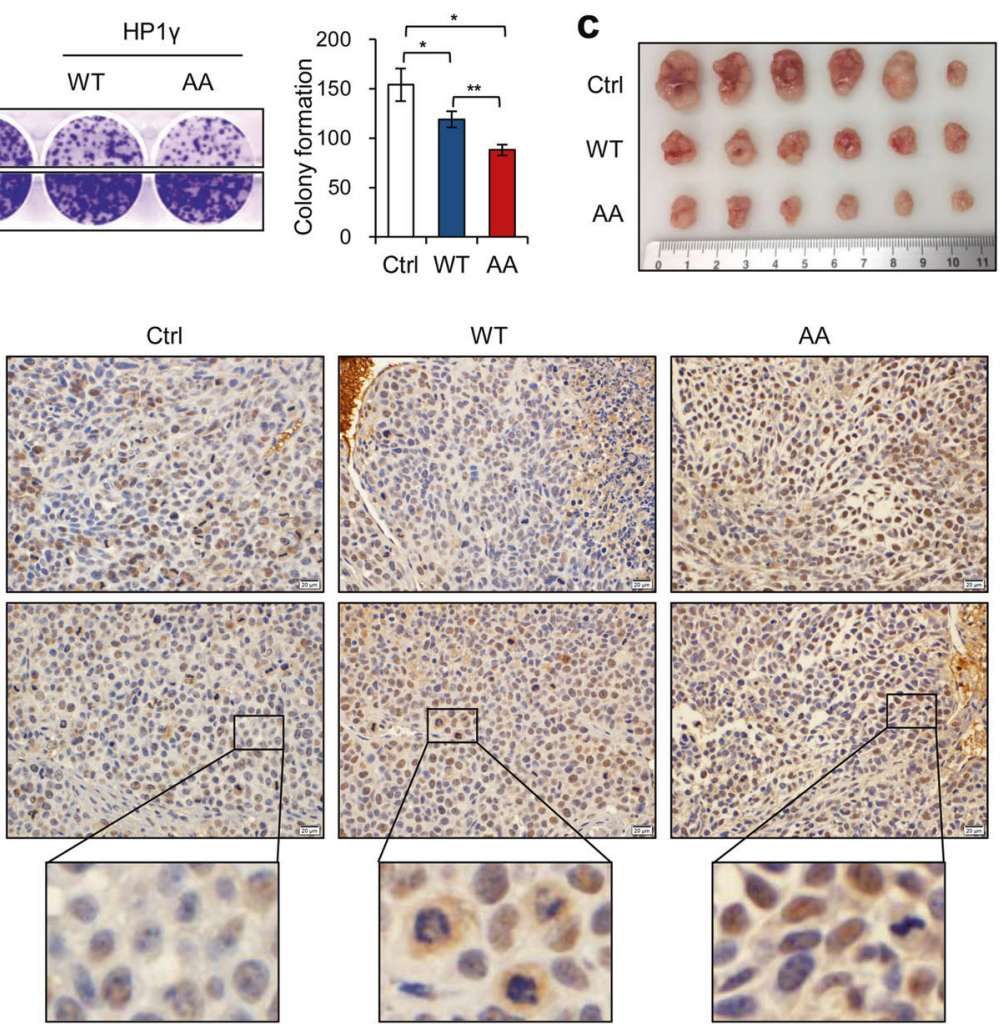

Fig. 3 Inhibiting the nuclear export of HP1 $\gamma$ promotes p53 effects in cervical cancer cells. a Immunoblot analysis of $\mathrm{SiHa}$ cells expressing doxycycline-induced HP1 WT or AA treated with or without doxycycline for $48 \mathrm{~h}$. b Clonogenic assay of $\mathrm{SiHa}$ cells expressing doxycycline-induced HP1 WT or AA treated with or without doxycycline for 20 days. c-e In vivo tumorigenesis assay of

unidentified role of HP1 $\gamma$ in the nucleus to prevent tumor development.

\section{UBE2L3 is a candidate target gene of HP1 isoforms in cervical cancer cells}

Based on the altered subcellular localization of HP1 $\gamma$ in cervical cancer, we reasoned that there would be a variety of changes in gene expression, under the control of HP1 $\gamma$. Thus, we performed a genome-wide microarray analysis of HP1-depleted HeLa cells (Supplementary Fig. S5, Supplementary Tables S1-S7). Among the candidate target genes commonly modulated by the three HP1 isoforms (Supplementary Table S1), UBE2L3 is known to be involved in proteasomal degradation of p53 during cervical cancer progression, interacting with E6AP to form ubiquitination machinery (Fig. 4a) [34, 35]. Indeed, we observed suppressed UBE2L3 expression and enhanced p53 signaling upon knockdown of HPV E6 (Supplementary Fig. S6). Depletion of UBE2L3 elevated p53 signaling, accordingly reducing cell growth rate and colony forming ability of cervical cancer cells (Supplementary Fig. S7). Analysis of GEO profiles (GDS2416) in 33 different biopsies from 16
$\mathrm{SiHa}$ cells was performed. SiHa cells expressing HP1 WT or AA were injected into the flank of nude mice. f Immunohistochemical staining of $\mathrm{HP} 1 \gamma$ protein in tumor tissues derived from mice injected with $\mathrm{SiHa}$ cells expressing HP1 WT or AA. Data are presented as the mean \pm SD $(n=6)$. $* P<0.05 ; * * P<0.01 ; * * * P<0.001$.

cervical cancer patients showed a negative correlation between the expression of $U B E 2 L 3$ and $C B X 3$ (a gene encoding HP1 $\gamma$ ), whereas the expression of $U B E 2 L 3$ and $C B X 1$ (a gene encoding HP1 $\beta$ ) showed a positive correlation (Fig. 4b). There was no statistically significant correlation between $U B E 2 L 3$ and $C B X 5$ (a gene encoding HP1 $\alpha$ ) levels (Fig. 4b). None of the three HP1 isoforms showed a correlation with the expression of $U B E 3 A$, a gene coding E6AP (Supplementary Fig. S8). The ChIP-qPCR analysis showed that the three HP1 isoforms were differentially recruited to the promoter of $U B E 2 L 3$ as well as other candidate target genes (Supplementary Fig. S5D, E), indicating that $U B E 2 L 3$ is a direct target of the HP1 isoforms. In addition, the protein level of UBE2L3 was elevated in HP1 $\gamma$ knockdown cells, whereas the depletion of HP1 $\beta$ led to an unexpected reduction of UBE2L3 protein (Fig. 4c). Consequently, the protein level of p53 exhibited an inverse correlation with the UBE2L3 level (Fig. 4c), which was resulted from alterations in p53 protein stability (Fig. 4d). None of the HP1 $\gamma$ or HP1 $\beta$ knockdown affected the TP53 mRNA level (Fig. 4e), demonstrating that the change in p53 protein level upon HP1 depletion did not originate from the regulation of its transcription. Moreover, HP1 $\gamma$ depletion 

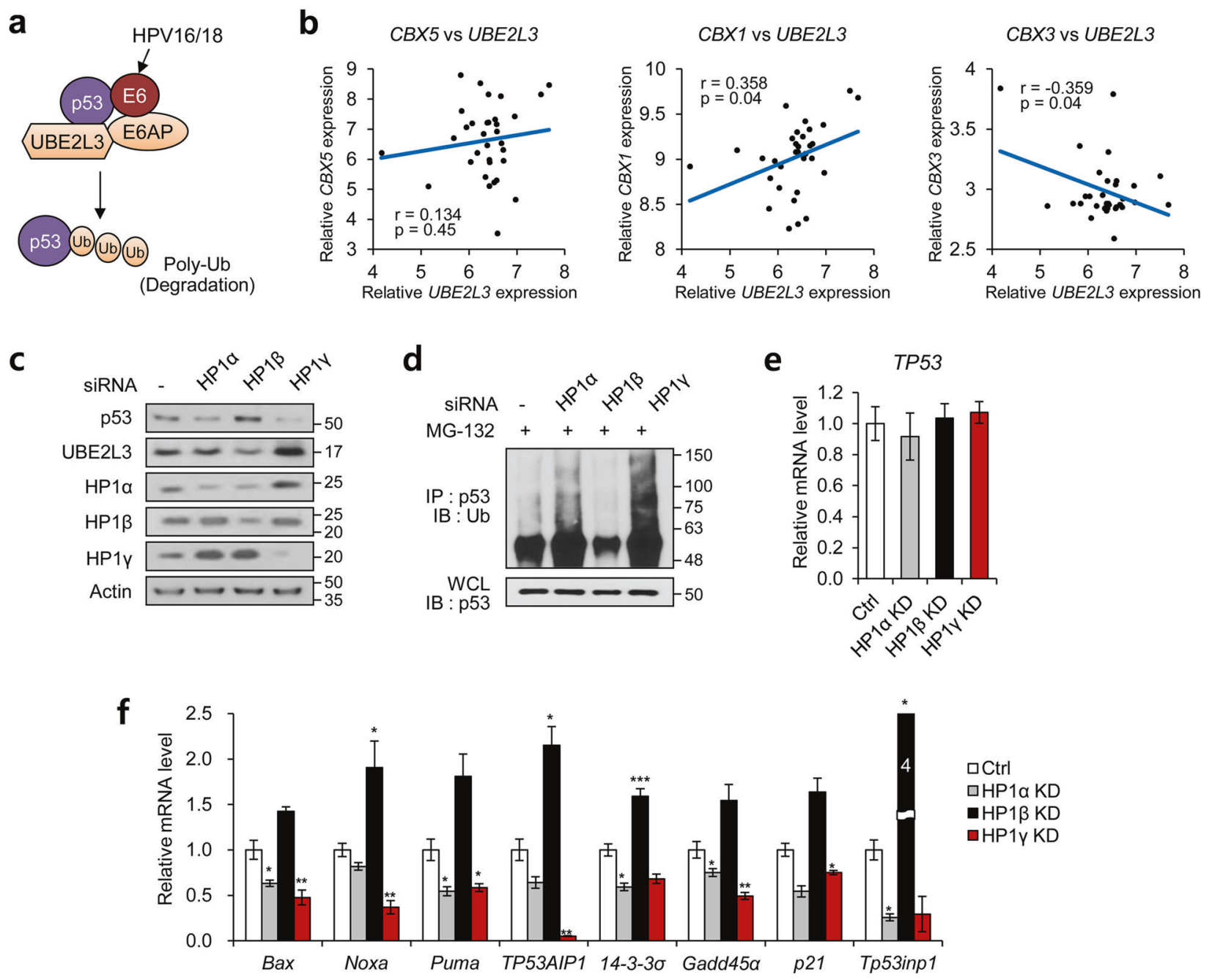

Fig. 4 HP1 isoforms regulate UBE2L3-dependent p53 ubiquitination in cervical cancer cells. a Model for UBE2L3-mediated p53 degradation upon high-risk HPV infection. b Pearson's correlation analysis in different areas of cervical cancer tumors (GDS2416) $(n=$ 33). c Immunoblot analysis of HeLa cells expressing siRNA of HP1 isoforms. d Immunoblot analysis of p53 immunoprecipitates (IP) and whole-cell lysates (WCL) from HeLa cells expressing siRNA of HP1 isoforms after MG-132 treatment. e The mRNA levels of TP53 in HeLa cells expressing siRNA of HP1 isoforms. $\mathbf{f}$ The mRNA levels of p53 target genes in HeLa cells expressing siRNA of HP1 isoforms. Data are presented as the mean $\pm \operatorname{SEM}(n=3)$. $* P<0.05, * * P<0.01$, $* * * P<0.001$. downregulated expression of p53 target genes, whereas HP1 $\beta$ depletion produced the opposite effect (Fig. 4f). The effects of HP1 $\alpha$ depletion were similar to but weaker than those of HP1 $\gamma$ depletion. These results indicate that HP1 isoforms differentially regulate the transcription of the $U B E 2 L 3$ gene.

\section{HP1 $\gamma$ is a major regulator of UBE2L3-dependent p53 ubiquitination}

As the depletion of each HP1 isoform led to a reciprocal increase in the expression of other HP1 isoforms (Fig. 4c), we hypothesized that the reduction in the UBE2L3 protein level by HP1 $\beta$ depletion would be produced through a compensatory response by HP1 $\alpha$ or HP1 $\gamma$. To test this, we examined the effect of co-silencing of HP1 $\beta$ with another HP1 isoform, HP1 $\alpha$ or HP1 $\gamma$, on the UBE2L3 and p53 protein levels. A reduction in UBE2L3 expression by HP1 $\beta$ depletion was recovered by co-knockdown of HP1 $\gamma$, but not HP1 $\alpha$ (Fig. 5a, b), further leading to the reduction in p53 protein level that was elevated by depletion of HP1 $\beta$ alone (Fig. 5a). This effect was mediated by the alteration of ubiquitination, but not the mRNA level of TP53 (Fig. 5c, d). The elevation of the expressions of p53 target genes by HP1 $\beta$ knockdown was also antagonized by HP1 $\gamma$ coknockdown (Fig. 5e). Critically, HP1 $\beta$ depletion significantly increased the recruitment of HP1 $\gamma$, but not HP1 $\alpha$, to the promoter of $U B E 2 L 3$ (Fig. 5f), indicating that the upregulation of the $\mathrm{p} 53$ protein by HP $1 \beta$ depletion was due to the suppression of $U B E 2 L 3$ expression through 
a

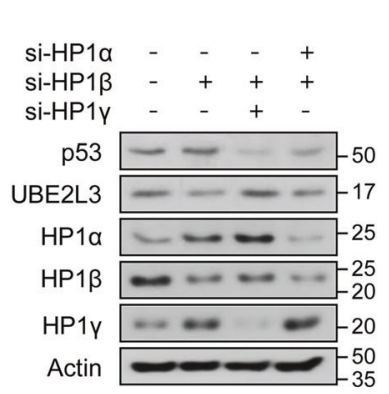

b

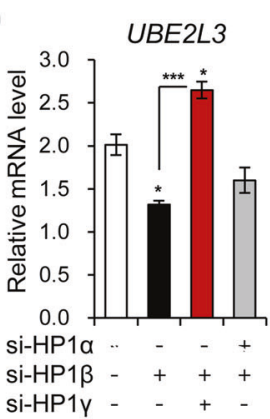

C

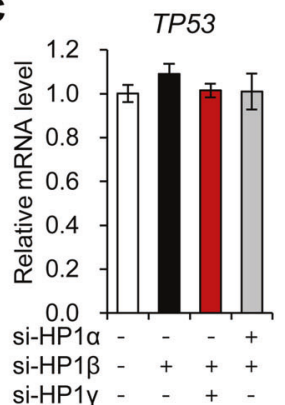

d

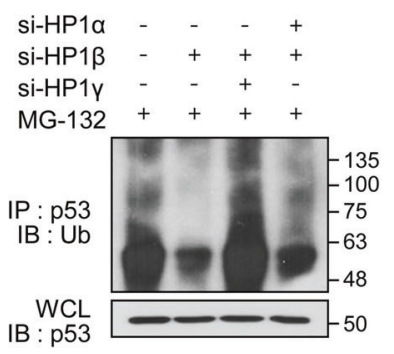

$\mathbf{f}$

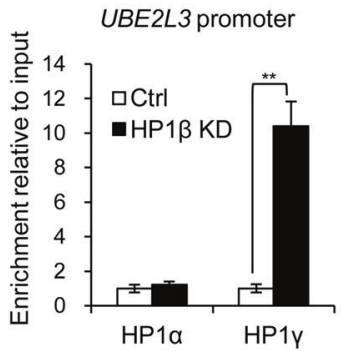

e

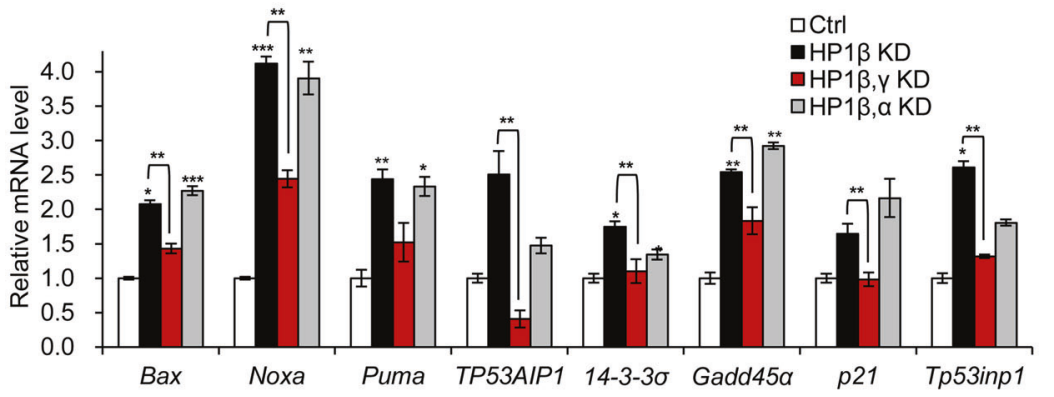

g

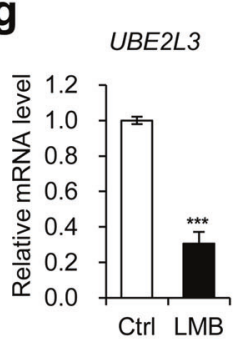

h

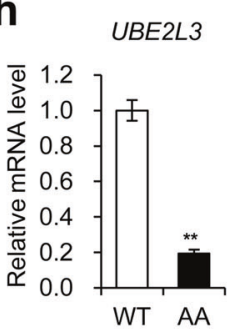

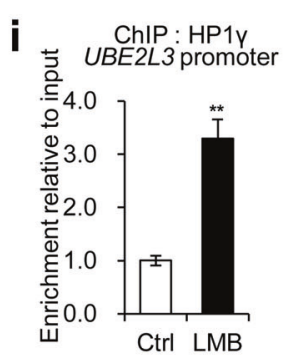

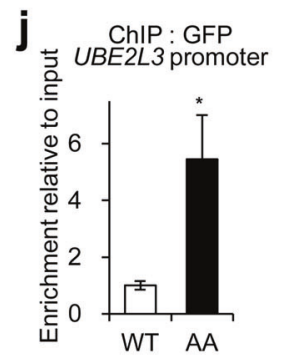

k

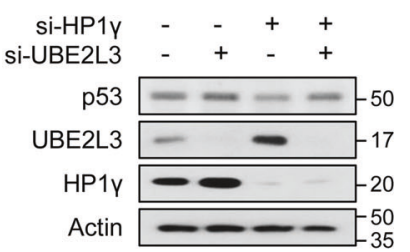

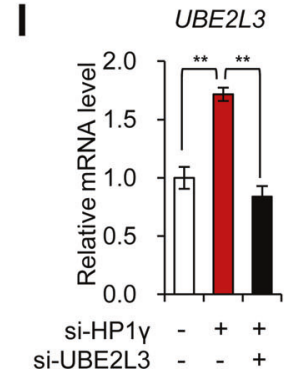

Fig. 5 HP1 $\gamma$ negatively regulates p53 ubiquitination by suppressing $U B E 2 L 3$ expression. a Immunoblot analysis of HeLa cells coexpressing siRNA of HP1 isoforms. b The mRNA levels of UBE2L3 in HeLa cells co-expressing siRNA of HP1 isoforms. c The mRNA levels of TP53 in HeLa cells co-expressing siRNA of HP1 isoforms. d Immunoblot analysis of p53 immunoprecipitates (IP) and whole-cell lysates (WCL) from HeLa cells co-expressing siRNA of HP1 isoforms after MG-132 treatment. e The mRNA levels of p53 target genes in HeLa cells co-expressing siRNA of HP1 isoforms. f HeLa cells were transfected with siRNA of HP1 $\beta$, followed by ChIP-qPCR analysis for HP1 $\alpha$ and HP1 $\gamma$ antibodies in the promoter region of the UBE2L3 gene. $\mathrm{g} \mathrm{SiHa}$ cells were treated with or without LMB, followed by ChIP-qPCR analysis with an HP1 $\gamma$ antibody in the promoter region of

compensation by HP1 $\gamma$. Indeed, inhibiting nuclear export of HP1 $\gamma$ using LMB or AA mutant successfully suppressed UBE2L3 expression (Fig. $5 \mathrm{~g}, \mathrm{~h}$ ) by recruitment of HP1 $\gamma$ to

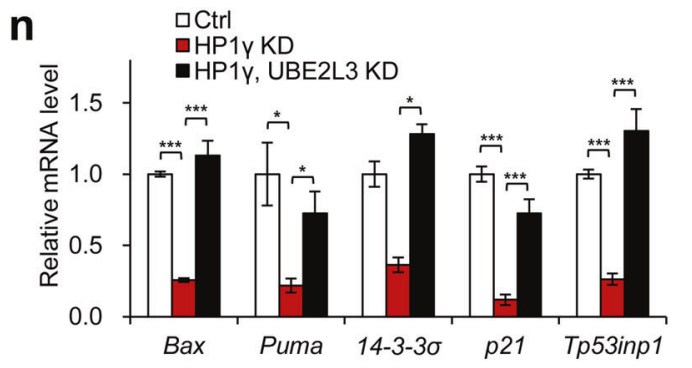

the UBE2L3 gene. $\mathbf{h}$ The mRNA levels of UBE2L3 in SiHa cells treated with or without LMB. i SiHa cells were transfected with GFPHP1 $\gamma$ WT or AA mutant vectors, followed by ChIP-qPCR analysis with a GFP antibody in the promoter region of $U B E 2 L 3$ gene. $\mathbf{j}$ The mRNA levels of $U B E 2 L 3$ in SiHa cells expressing GFP-HP1 $\gamma$ WT or AA mutant. k Immunoblot analysis of HeLa cells co-expressing siRNA of HP1 $\gamma$ and UBE2L3. I The mRNA levels of UBE2L3 in HeLa cells co-expressing siRNA of HP1 $\gamma$ and UBE2L3. m The mRNA levels of TP53 in HeLa cells co-expressing siRNA of HP1 $\gamma$ and UBE2L3. $\mathbf{n}$ The mRNA levels of p53 target genes in HeLa cells co-expressing siRNA of HP1 $\gamma$ and UBE2L3. Data are presented as the mean $\pm \operatorname{SEM}(n=3) . * P<0.05, * * P<0.01, * * * P<0.001$.

the $U B E 2 L 3$ promoter (Fig. $5 \mathrm{i}, \mathrm{j}$ ). In contrast, knockdown of HP1 $\alpha$ did not lead to compensatory recruitment of HP1 $\gamma$ to the promoter of UBE2L3 (Supplementary Fig. S9A). 
To explore whether the upregulation of p53 by HP1 $\gamma$ is dependent on UBE2L3 suppression, we observed the effects of HP1 $\gamma$ knockdown in UBE2L3-depleted HeLa cells (Fig. 5k, 1). The reduction of p53 protein level by HP1 $\gamma$ knockdown was reversed by the co-silencing of UBE2L3, without changing mRNA level of TP53 (Fig. 5k, m), indicating that the suppression of $U B E 2 L 3$ expression is required for $\mathrm{HP} 1 \gamma$-mediated upregulation of p53. Consistently, decreased expressions of p53 target genes upon HP1 $\gamma$ knockdown were recovered by the co-knockdown of UBE2L3 (Fig. 5n). However, the co-knockdown of HP1 $\alpha$ and UBE2L3 partially recovered HP1 $\alpha$ knockdownmediated inhibition of p53 signaling (Supplementary Fig. S9B, C).

To investigate whether HP1s-mediated UBE2L3 alteration is dependent on HPV E6, we tested the effects of ectopic expression of HPV16 E6 in HPV-negative cancer cells (U2OS). Although E6 expression in U2OS cells lowered the level of HP1 $\alpha$ and HP1 $\beta$ while upregulating HP1 $\gamma$ expression (Supplementary Fig. S10A, B), enrichment of HP1 $\gamma$ on UBE2L3 promoter significantly decreased (Supplementary Fig. S10C). Taken together, these data demonstrate that HP1 $\gamma$ mainly suppresses $U B E 2 L 3$ expression to elevate p53 signaling, which is dependent on HPV E6.

\section{Overexpression of HP1 $\gamma$ promotes p53 effects by directly suppressing UBE2L3 expression in an HPV- positive manner}

Next, we assessed whether HP1 $\gamma$ overexpression prevents UBE2L3-mediated p53 degradation. Overexpression of HP1 $\gamma$ in HeLa cells blocked the expression of UBE2L3 (Fig. 6a, b) and ubiquitination of p53 (Fig. 6c), resulting in an increase in p53 protein level (Fig. 6a) but not TP53 mRNA (Fig. 6d). Moreover, p53 was significantly recruited to the promoters of its target genes (Fig. 6e), leading to the upregulation of their expression (Fig. 6f). In addition, ectopic expression of HP1 $\gamma$ induced the expression of apoptotic markers (Fig. 6g) and reduced the growth rate of HeLa cells (Fig. 6h). The positive effects of HP1 $\gamma$ overexpression on p53 function were further confirmed by similar observations in other HPV-positive cervical cancer cell lines, SiHa, and CaSki (Supplementary Fig. S11). However, in HPV-negative cell lines (C33A HPV-negative cervical cancer cells and U2OS osteosarcoma cells), HP1 $\gamma$ overexpression had no effect on the protein levels of UBE2L3 and p53 (Supplementary Fig. S12A) as well as the expression of p53 target genes (Supplementary Fig. S12B). Interestingly, HP1 $\gamma$ overexpression in C33A and U2OS cells ectopically expressing HPV16 E6 produced similar effects observed in HPV-positive cervical cancer cells (Supplementary Fig. S12C, D). These data indicate that the role of ectopically expressed $\mathrm{HP} 1 \gamma$ in p53 elevation is specific for high-risk HPV.

Next, we checked whether overexpression of HP1 $\beta$ affects the regulatory function of $\mathrm{HP} 1 \gamma$ in suppression of UBE2L3. Overexpression of HP1 $\beta$ increased the level of p53 and its target genes (Supplementary Fig. S13A, B), but UBE2L3 level was not altered by HP1 $\beta$ overexpression (Supplementary Fig. S13A, C). There was a slight synergy effect by co-overexpressing HP1 $\beta$ and HP1 $\gamma$ on induction of p53 signaling and inhibition of colony formation of cervical cancer cells (Supplementary Fig. S13A, B, D), while UBE2L3 suppression by HP1 $\gamma$ was not affected by co-expression of HP1 $\beta$ (Supplementary Fig. S13C). These data suggest that HP1 $\beta$ induces p53 function through an independent mechanism of UBE2L3.

Since we observed that HP1 $\gamma$ recruited to the promoter region of the UBE2L3 gene (Fig. 5F), we tested whether chromatin binding of HP1 $\gamma$ is critical for the suppression of UBE2L3 expression. We generated an HP1 $\gamma$ CD mutant possessing a single-site mutation from valine 32 (V32) to alanine (Fig. 6i), which leads to a failure in chromatin binding [36]. As expected, HP1 $\gamma \mathrm{V} 32 \mathrm{~A}$ was not recruited to the promoter region of $U B E 2 L 3$, whereas ectopically expressed WT HP1 $\gamma$ were enriched on the promoter region (Fig. 6j). Unlike WT HP1 $\gamma$, HP1 $\gamma$ V32A failed to suppress UBE2L3 expression (Fig. 6k, 1), elevate the p53 protein level (Fig. 61), and promote transcription of p53 target genes (Fig. 6m). These data suggest that the binding of HP1 $\gamma$ to the promoter region of the UBE2L3 gene is required for suppression of the gene and subsequent induction of p53 signaling.

To further examine the functional involvement of the CSD in this response, we also produced two CSD mutants of HP1 $\gamma$ (Fig. 6i). The HP1 $\gamma$ I165E mutant disrupts homodimerization, while the HP1 $\gamma$ W174A mutant fails to interact with other proteins containing the PXVXL motif [19, 37]. Despite substantial chromatin binding of both HP1 $\gamma$ CSD mutants (Fig. 6j), neither HP1 $\gamma$ I165E nor HP1 $\gamma$ W174A suppressed UBE2L3 expression, elevated the p53 protein level (Supplementary Fig. S14A-C), and reduced polyubiquitination of p53 (Supplementary Fig. S14D). Consistently, both mutants were unable to induce apoptotic markers (Supplementary Fig. S14A), expression of p53 target genes (Supplementary Fig. S14E), and the growth arrest of cervical cancer cells (Supplementary Fig. $\mathrm{S} 14 \mathrm{~F})$. In addition, flow cytometric analysis showed an increase in the proportion of apoptotic cells among cells expressing WT but not mutants HP1 $\gamma$ (Supplementary Fig. S14G-I). Taken together, these results indicate that all the domains of HP1 $\gamma$ are required for the suppression of $U B E 2 L 3$ expression and subsequent upregulation of $\mathrm{p} 53$. 


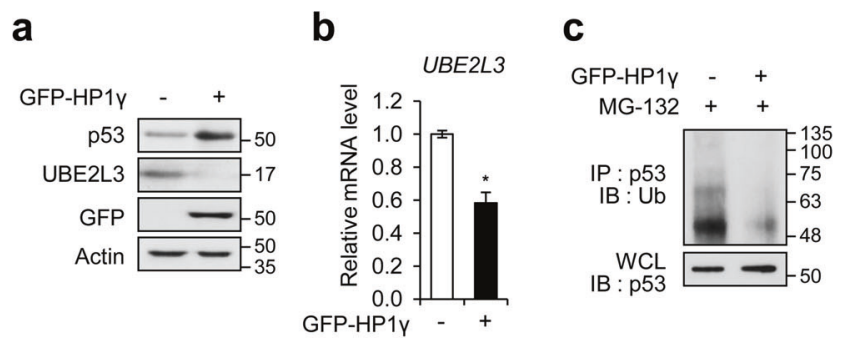

f

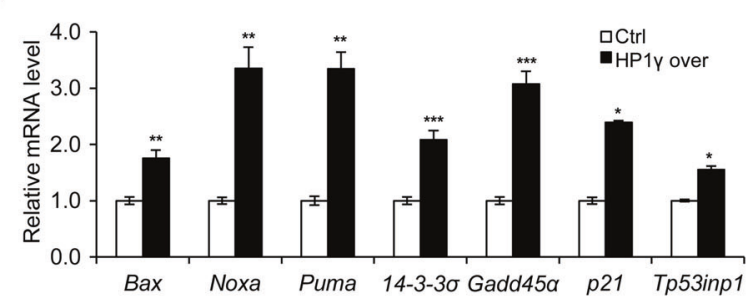

d

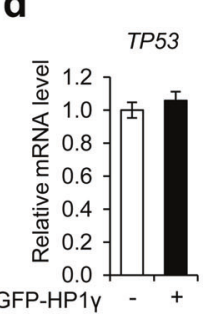

e

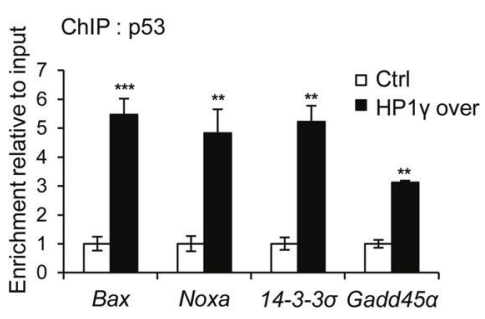

g

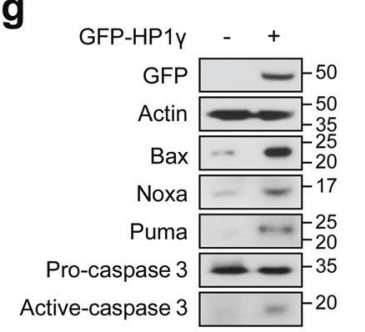

h

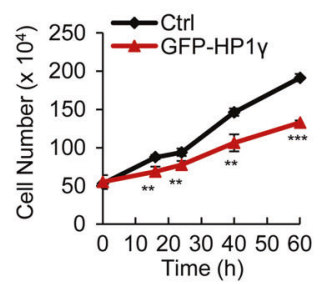

i

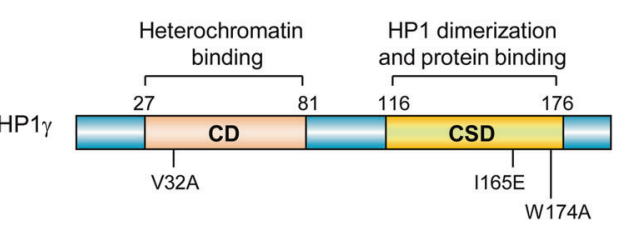

j

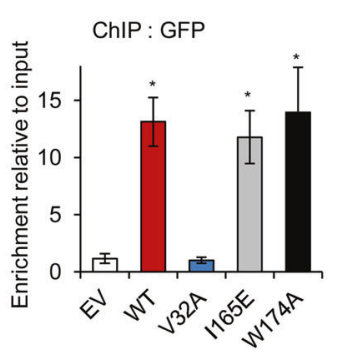

k
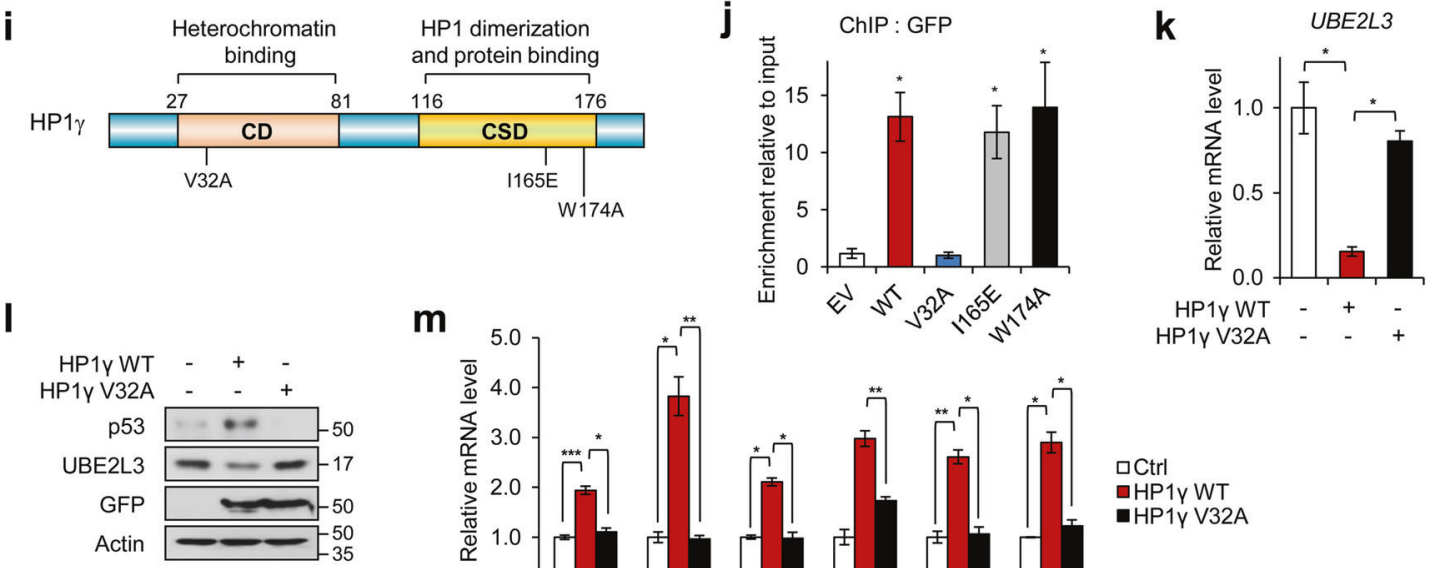

m

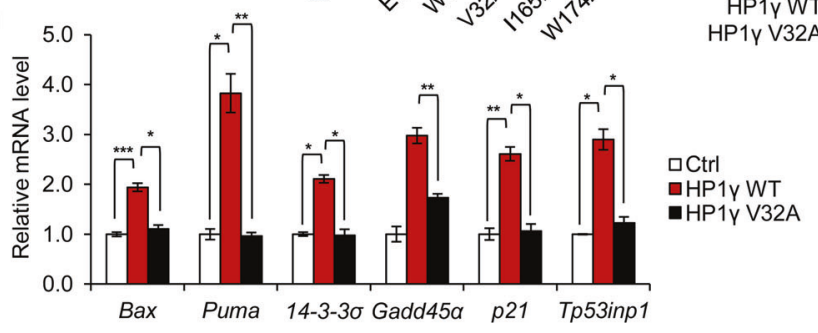

Fig. 6 Overexpression of HP1 $\gamma$ promotes p53 signaling by directly suppressing $\boldsymbol{U B E 2 L 3}$ expression. a Immunoblot analysis of HeLa cells expressing GFP-HP1 $\gamma$. b The mRNA levels of UBE2L3 in HeLa cells expressing GFP-HP1 $\gamma$. c Immunoblot analysis of p53 immunoprecipitates (IP) and whole-cell lysates (WCL) from HeLa cells expressing GFP-HP1 $\gamma$ after MG-132 treatment. d The mRNA levels of TP53 in HeLa cells expressing GFP-HP1 $\gamma$. e ChIP-qPCR analysis with a p53 antibody in the promoter regions of p53 target genes in HeLa cells expressing GFP-HP1 $\gamma$. f The mRNA levels of p53 target genes in $\mathrm{HeLa}$ cells expressing GFP-HP1 $\gamma$. g Immunoblot analysis of HeLa cells expressing GFP-HP1 $\gamma$. h The growth rate of HeLa expressing

GFP-HP1 $\gamma$. i Schematic representation of HP1 $\gamma$ protein and partial CD sequence containing V32. j HeLa cells were transfected with GFPHP1 $\gamma$ WT or mutant (V32A, I165E, or W174A) vectors, followed by ChIP-qPCR analysis with a GFP antibody in the promoter regions of the UBE2L3 gene. $\mathbf{k}$ The mRNA levels of UBE2L3 in HeLa cells expressing GFP-HP1 $\gamma$ wild-type (WT) or V32A mutant. 1 Immunoblot analysis of HeLa cells expressing GFP-HP1 $\gamma$ WT or V32A mutant. m The mRNA levels of p53 target genes in HeLa cells expressing GFP-HP1 $\gamma$ WT or V32A mutant. Data are presented as the mean \pm SEM $(n=3) . * P<0.05 ; * * P<0.01 ; * * * P<0.001$.

\section{Discussion}

The role of HP1 $\gamma$ in euchromatic gene regulation as well as in heterochromatic formation has been suggested, because of its unique subnuclear localization $[15,23]$. Based on the hypothesis that the three HP1 isoforms differentially regulate gene expression, we explored the effects of HP1 depletion on gene regulation throughout the human genome. Within the commonly regulated genes, we identified an interesting target gene, $U B E 2 L 3$, which contributes to HPV-mediated proteasomal degradation of p53. Despite the

overlapping gene regulation in the microarray screening, our data demonstrate that HP1 $\gamma$ is a key suppressor of UBE2L3 expression (Fig. 4). Although HP1 $\alpha$ and HP1 $\beta$ also increased p53 level, they were not closely related to HPV E6-mediated UBE2L3 induction or HP1 $\gamma$-mediated UBE2L3 suppression. The regulation of $\mathrm{p} 53$ by HP1 $\alpha$ was partially dependent on UBE2L3 (Supplementary Fig. S9), while HP1 $\beta$ seemed to regulate p53 through a separate mechanism from UBE2L3 (Supplementary Fig. S10). These results suggest that the involvement of each HP1 isoform in UBE2L3-p53 axis is different. A previous study has 
a High-risk HPV-positive cervical cancer

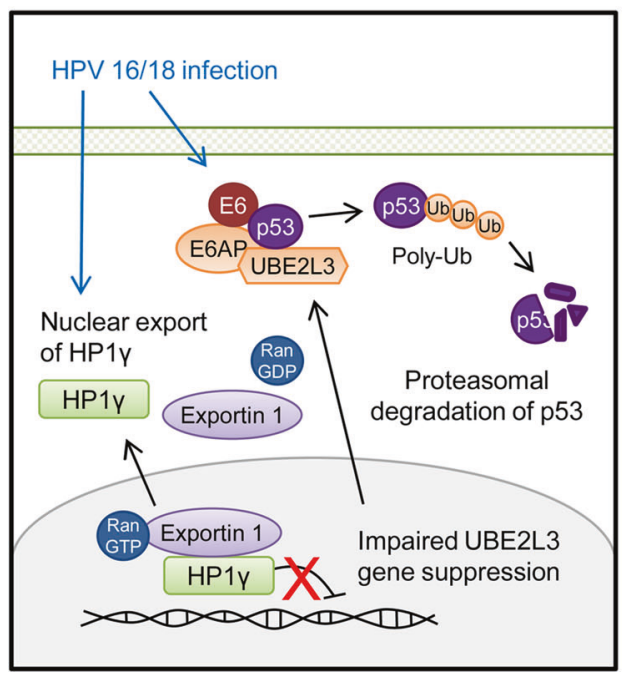

Fig. 7 Molecular model describing p53 regulation by HP1 $\gamma$ dependent UBE2L3 suppression. a Upon high-risk HPV infection, the E6 oncoprotein of HPV interacts with p53 and E6AP to induce polyubiquitination and proteasomal degradation of p53. Moreover, HPV infection induces exportin-1-mediated nuclear export of HP1 $\gamma$, thereby allowing plenty of UBE2L3 to be expressed, which results in

revealed that the length of the hinge region connecting $\mathrm{CD}$ and CSD determines the distinct binding properties of HP1 isoforms [38]. Thus, a relatively short hinge region of $\mathrm{HP} 1 \gamma$ compared with that of HP1 $\alpha$ and HP1 $\beta$ appears to be con-

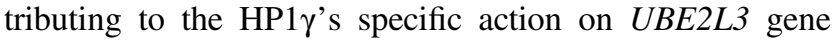
regulation.

Many diseases are associated with a dysfunction of ubiquitin signaling, with the E3 ligases being the focus. However, recent evidence demonstrates that mutations or impairments of the E2-conjugating enzymes can lead to severe diseases [39]. Given their relevance to diseases, E2conjugating enzymes may serve as an important family of therapeutic targets. The functional roles of several E2s in carcinogenesis have been reported [40]. UBE2L3 is one of the most abundant E2s in mammalian cell lines [41] and associated with diverse cancers including hepatocellular carcinoma [42], oral cancer [43], prostate cancer [44], and non-small cell lung cancer [45]. Our findings show that $U B E 3 L 3$ is transcriptionally repressed by $\mathrm{HP} 1 \gamma$, which is impaired in cervical cancer cells due to the nuclear export of HP1 $\gamma$. Hence, UBE2L3 might be a potential therapeutic target to treat cancers that are characterized by elevated UBE2L3 expression.

During the development of cervical cancer, oncogenic HPV E6 plays a central role by disabling the tumorsuppressing function of p53 [11]. In this process, E6AP interacts with UBE2L3, and this complex participates in p53 ubiquitination [10, 12]. Hence, inhibiting the E6mediated degradation of $\mathrm{p} 53$ could be a potent strategy to b Excessive nuclear HP1y in cervical cancer

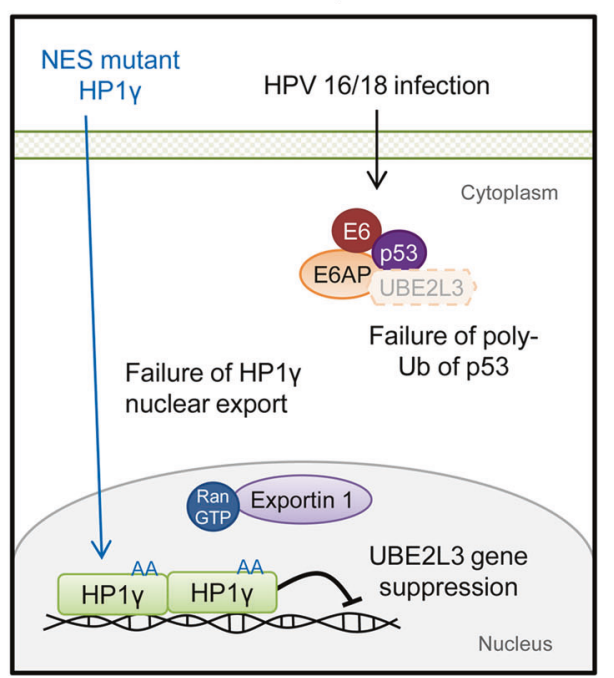

p53 degradation in an HPV-specific manner. b When the NES mutant $\mathrm{HP} 1 \gamma$ (HP1 $\gamma \mathrm{AA})$ is overexpressed artificially in cervical cancer cells, exportin-1 fails to transport HP1 $\gamma$ AA to the cytoplasm and HPVspecific degradation of p53 is impaired due to UBE2L3 suppression by nuclear HP1 $\gamma$.

combat cervical cancer. Recent studies have identified several strategies to inactivate E6 and E7 using RNAi, TALEN, or CRISPR-mediated gene disruption [46-49] or small molecules [50, 51]. However, the previous strategies were limited to in vitro and mouse models and have not yet been tested in human trials [46-51]. In this current study, we present a new approach to protect p53 from E6-mediated degradation by controlling UBE2L3 expression with HP1 $\gamma$. HP1 $\gamma$ negatively regulates the ubiquitin-proteasome system (UPS)-dependent p53 degradation through the transcriptional repression of UBE2L3 while increasing overall p53 protein levels and restoring p53 function (Fig. 7). Intriguingly, these effects of HP1 $\gamma$ were observed only in HPVpositive cervical cancer cells but not HPV-negative cancer cells. Considering that E6AP, rather than MDM2, is a major E3 ubiquitin ligase in HPV-infected cervical cancer cells [52], the effects of HP1 $\gamma$ we observed here was most likely due to suppression of UBE2L3-E6AP-p53 UPS. In line with the previous finding, HPV-positive cervical cancer cells eventually died after overexpression of $\mathrm{HP} 1 \gamma$, suggesting that even a partial rescue of p53 is sufficient to induce apoptosis.

Another important issue we found here is the novel role of high-risk HPV E6 in cervical cancer progression through the exportin-1-mediated nuclear export of HP1 $\gamma$. Although a few papers have proposed the presence of HP1 $\gamma$ in the cytoplasm during maternal to embryonic transition [53] or in myoblasts [54], disease-specific subcellular migration of HP1 $\gamma$ has not been reported yet. In the case of cervical 
cancer cells, HPV E6 utilizes various Karyopherin proteins for nuclear export and import, after which it can interact with nuclear transcription factors such as p53 [55]. The stability and nuclear export of p53 have been reported to rely on chromosomal regional maintenance-1/exportin-1 activity [29], which is promoted by high-risk HPV E6 [30]. Therefore, exportin-1 is very critical for cancer cell survival and high expression of exportin-1 has been reported in cervical cancer [56]. Interestingly, we found abnormal cytoplasmic localization of $\mathrm{HP} 1 \gamma$, which results from increased binding with exportin-1, in HPV-infected cervical cancer cells and tissues. Since HP1 $\gamma$ is observed only within the nucleus in the precancer stage, chronic leakage of HP1 $\gamma$ to the cytoplasm upon persistent infection with high-risk HPV might be an important step in the cancer progression of HPV-infected cells. Notably, inhibition of the nuclear export of HP1 $\gamma$ rescued the p53 level and suppressed tumor growth. These findings suggest that the enhancement of nuclear retention of $\mathrm{HP} 1 \gamma$ by pharmacological intervention would offer a potential therapeutic strategy to overcome HPV-specific p53 degradation in cervical cancer.

Acknowledgements Raw data have been deposited in Gene Expression Omnibus (GEO) profiles (accession no. GDS2416 and GDS3233). This research was supported by the National Research Foundation of Korea (NRF) funded by the Ministry of Science, ICT \& Future Planning (2017R1A6A3A04001986 to SAY, 2017R1A2B3002186 and 2019R1A5A2027340 to JWH, 2018R1A6A1A03023718 and 2019R1A2C1008619 to SHK).

\section{Compliance with ethical standards}

Conflict of interest The authors declare that they have no conflict of interest.

Publisher's note Springer Nature remains neutral with regard to jurisdictional claims in published maps and institutional affiliations.

Open Access This article is licensed under a Creative Commons Attribution 4.0 International License, which permits use, sharing, adaptation, distribution and reproduction in any medium or format, as long as you give appropriate credit to the original author(s) and the source, provide a link to the Creative Commons license, and indicate if changes were made. The images or other third party material in this article are included in the article's Creative Commons license, unless indicated otherwise in a credit line to the material. If material is not included in the article's Creative Commons license and your intended use is not permitted by statutory regulation or exceeds the permitted use, you will need to obtain permission directly from the copyright holder. To view a copy of this license, visit http://creativecommons. org/licenses/by/4.0/.

\section{References}

1. Holowaty P, Miller AB, Rohan T, To T. Natural dysplasia of the uterine cervix. J Nat Cancer Inst. 1999;91:252-8.

2. Dürst M, Gissmann L, Ikenberg H, zur Hausen H. A papillomavirus DNA from a cervical carcinoma and its pre valence in cancer biopsy samples from different geographic regions. Proc Natl Acad Sci USA. 1983;80:3812-5.

3. Smith JS, Lindsay L, Hoots B, Keys J, Franceschi S, Winer R, et al. Human papillomavirus type distribution in invasive cervical cancer and high-grade cervical lesions: a meta-analysis update. Int J Cancer. 2007;121:621-32.

4. Hildesheim A, Herrero R, Wacholder S, Rodriguez AC, Solomon D, Bratti MC, et al. Effect of human papillomavirus 16/18 L1 viruslike particle vaccine among young women with preexisting infection: a randomized trial. JAMA. 2007;298:743-53.

5. Moore DH, Tian C, Monk BJ, Long HJ, Omura GA, Bloss JD. Prognostic factors for response to cisplatin-based chemotherapy in advanced cervical carcinoma: a Gynecologic Oncology Group Study. Gynecol Oncol 2010;116:44-9.

6. Wolford JE, Tewari KS. Rational design for cervical cancer therapeutics: cellular and non-cellular based strategies on the horizon for recurrent, metastatic or refractory cervical cancer. Expert Opin Drug Discov. 2018;13:445-57.

7. von Knebel Doeberitz M, Rittmüller C, zur Hausen H, Dürst M. Inhibition of tumorigenicity of cervical cancer cells in nude mice by HPV E6-E7 anti-sense RNA. Int J Cancer. 1992;51:831-4.

8. Dyson N, Howley PM, Münger K, Harlow E. The human papilloma virus-16 E7 oncoprotein is able to bind to the retinoblastoma gene product. Science. 1989;243:934-7.

9. Scheffner M, Werness BA, Huibregtse JM, Levine AJ, Howley PM. The E6 oncoprotein encoded by human papillomavirus types 16 and 18 promotes the degradation of p53. Cell. 1990;63:1129-36.

10. Werness BA, Levine AJ, Howley PM. Association of human papillomavirus types 16 and 18 E6 proteins with p53. Science. 1990;248:76-9.

11. Martinez-Zapien D, Ruiz FX, Poirson J, Mitschler A, Ramirez J, Forster A, et al. Structure of the E6/E6AP/p53 complex required for HPV-mediated degradation of p53. Nature. 2016;529:541-5.

12. Vogt M, Butz K, Dymalla S, Semzow J, Hoppe-Seyler F. Inhibition of Bax activity is crucial for the antiapoptotic function of the human papillomavirus E6 oncoprotein. Oncogene. 2006;25:4009-15.

13. Bannister AJ, Zegerman P, Partridge JF, Miska EA, Thomas JO, Allshire RC, et al. Selective recognition of methylated lysine 9 on histone H3 by the HP1 chromo domain. Nature. 2001;410:120-4.

14. Nielsen PR, Nietlispach D, Mott HR, Callaghan J, Bannister A, Kouzarides T, et al. Structure of the HP1 chromodomain bound to histone H3 methylated at lysine 9. Nature. 2002;416:103-7.

15. Nielsen AL, Oulad-Abdelghani M, Ortiz JA, Remboutsika E, Chambon P, Losson R. Heterochromatin formation in mammalian cells: interaction between histones and HP1 proteins. Mol Cell. 2001;7:729-39.

16. Zhang R, Adams PD. Heterochromatin and its relationship to cell senescence and cancer therapy. Cell Cycle. 2007;6:784-9.

17. Dialynas GK, Vitalini MW, Wallrath LL. Linking Heterochromatin Protein 1 (HP1) to cancer progression. Mutat Res. 2008;647:13-20.

18. Ayoub N, Jeyasekharan AD, Venkitaraman AR. Mobilization and recruitment of HP1: a bimodal response to DNA breakage. Cell Cycle. 2009;8:2945-50.

19. Brasher SV, Smith BO, Fogh RH, Nietlispach D, Thiru A, Nielsen PR, et al. The structure of mouse HP1 suggests a unique mode of single peptide recognition by the shadow chromo domain dimer. EMBO J. 2000;19:1587-97.

20. Nielsen SJ, Schneider R, Bauer UM, Bannister AJ, Morrison A, O'Carroll $\mathrm{D}$, et al. Rb targets histone $\mathrm{H} 3$ methylation and HP1 to promoters. Nature. 2001;412:561-5.

21. Jacobs SA, Khorasanizadeh S. Structure of HP1 chromodomain bound to a lysine 9-methylated histone H3 tail. Science. 2002;295:2080-3. 
22. Cowieson NP, Partridge JF, Allshire RC, McLaughlin PJ. Dimerisation of a chromo shadow domain and distinctions from the chromodomain as revealed by structural analysis. Curr Biol. 2000;10:517-25.

23. Minc E, Allory Y, Worman HJ, Courvalin JC, Buendia B. Localization and phosphorylation of HP1 proteins during the cell cycle in mammalian cells. Chromosoma. 1999;108:220-34.

24. De Koning L, Savignoni A, Boumendil C, Rehman H, Asselain B, Sastre-Garau X, et al. Heterochromatin protein 1alpha: a hallmark of cell proliferation relevant to clinical oncology. EMBO Mol Med. 2009;1:178-91.

25. Itsumi M, Shiota M, Yokomizo A, Kashiwagi E, Takeuchi A, Tatsugami K, et al. Human heterochromatin protein 1 isoforms regulate androgen receptor signaling in prostate cancer. $\mathrm{J}$ Mol Endocrinol. 2013;50:401-9.

26. Liu M, Huang F, Zhang D, Ju J, Wu XB, Wang Y, et al. Heterochromatin protein HP1 $\gamma$ promotes colorectal cancer progression and is regulated by miR-30a. Cancer Res. 2015;75:4593-5605.

27. Yi SA, Um SH, Lee J, Yoo JH, Bang SY, Park EK, et al. S6K1 phosphorylation of H2B mediates EZH2 trimethylation of H3: a determinant of early adipogenesis. Mol Cell. 2016;62:443-52.

28. Ryu HW, Shin DH, Lee DH, Choi J, Han G, Lee KY, et al. HDAC6 deacetylates p53 at lysines 381/382 and differentially coordinates p53-induced apoptosis. Cancer Lett. 2017;391:162-71.

29. Freedman DA, Levine AJ. Nuclear export is required for degradation of endogenous p53 by MDM2 and human papillomavirus E6. Mol Cell Biol. 1998;18:7288-93.

30. Stewart D, Ghosh A, Matlashewski G. Involvement of nuclear export in human papillomavirus type 18 E6-mediated ubiquitination and degradation of p53. J Virol. 2005;79:8773-83.

31. Koivusalo R, Mialon A, Pitkänen H, Westermarck J, Hietanen S. Activation of p53 in cervical cancer cells by human papillomavirus E6 RNA interference is transient, but can be sustained by inhibiting endogenous nuclear export-dependent p53 antagonists. Cancer Res. 2006;66:11817-24.

32. Hietanen S, Lain S, Krausz E, Blattner C, Lane DP. Activation of p53 in cervical carcinoma cells by small molecules. Proc Natl Acad Sci USA. 2000;97:8501-6.

33. Dong X, Biswas A, Süel KE, Jackson LK, Martinez R, Gu H. Structural basis for leucine-rich nuclear export signal recognition by CRM1. Nature. 2009;458:1136-41.

34. Scheffner M, Huibregtse JM, Vierstra RD, Howley PM. The HPV-16 E6 and E6-AP complex functions as a ubiquitin-protein ligase in the ubiquitination of p53. Cell. 1993;75:495-505.

35. Huang L, Kinnucan E, Wang G, Beaudenon S, Howley PM, Huibregtse JM, et al. Structure of an E6AP-UbcH7 complex: insights into ubiquitination by the E2-E3 enzyme cascade. Science. 1999;286:1321-6.

36. Choi JD, Park MA, Lee JS. Suppression and recovery of BRCA1mediated transcription by HP1 $\gamma$ via modulation of promoter occupancy. Nucleic Acids Res. 2012;40:11321-38.

37. Nozawa RS, Nagao K, Masuda HT, Iwasaki O, Hirota T, Nozaki $\mathrm{N}$. Human POGZ modulates dissociation of HP1alpha from mitotic chromosome arms through Aurora B activation. Nat Cell Biol. 2010;12:719-27.

38. Mishima Y, Jayasinghe $\mathrm{CD}, \mathrm{Lu} \mathrm{K}$, Otani J, Shirakawa M, Kawakami $\mathrm{T}$, et al. Nucleosome compaction facilitates HP1 $\gamma$ binding to methylated H3K9. Nucleic Acids Res. 2015;43:10200-12.
39. Alpi AF, Chaugule V, Walden H. Mechanism and disease association of E2-conjugating enzymes: lessons from UBE2T and UBE2L3. Biochem J. 2016;473:3401-19.

40. Zhou MJ, Chen FZ, Chen HC. Ubiquitination involved enzymes and cancer. Med Oncol. 2014;31:93.

41. Clague MJ, Heride C, Urbé $S$. The demographics of the ubiquitin system. Trends Cell Biol. 2015;25:417-26.

42. Iizuka $\mathrm{N}$, Tsunedomi $\mathrm{R}$, Tamesa $\mathrm{T}$, Okada $\mathrm{T}$, Sakamoto $\mathrm{K}$, Hamaguchi $\mathrm{T}$, et al. Involvement of c-myc-regulated genes in hepatocellular carcinoma related to genotype-C hepatitis B virus. J Cancer Res Clin Oncol. 2006;132:473-81.

43. Cheng LH. Ubiquitin and malignant transformation of oral mucosa. Head Neck. 2001;23:972-8.

44. Wang XS, Shankar S, Dhanasekaran SM, Ateeq B, Sasaki AT, Jing $\mathrm{X}$, et al. Characterization of KRAS rearrangements in metastatic prostate cancer. Cancer Discov. 2011;1:35-43.

45. Ma X, Zhao J, Yang F, Liu H, Qi W. Ubiquitin conjugating enzyme E2 L3 promoted tumor growth of NSCLC through accelerating p27kip1 ubiquitination and degradation. Oncotarget. 2017;8:84193-203.

46. Zhou J, Li B, Peng C, Wang F, Fu Z, Zhou C, et al. Inhibition of cervical cancer cell growth in vitro and in vivo by lentiviral-vector mediated shRNA targeting the common promoter of HPV16 E6 and E7 oncogenes. Antivir Res. 2013;98:305-13.

47. Kennedy EM, Kornepati AV, Goldstein M, Bogerd HP, Poling $\mathrm{BC}$, Whisnant $\mathrm{AW}$, et al. Inactivation of the human papillomavirus E6 or E7 gene in cervical carcinoma cells by using a bacterial CRISPR/Cas RNA-guided endonuclease. J Virol. 2014;88:11965-72.

48. Hu Z, Ding W, Zhu D, Yu L, Jiang X, Wang X, et al. TALENmediated targeting of HPV oncogenes ameliorates HPV-related cervical malignancy. J Clin Investig. 2015;125:425-36.

49. Nishida H, Matsumoto $Y$, Kawana K, Christie RJ, Naito M, Kim BS, et al. Systemic delivery of siRNA by actively targeted polyion complex micelles for silencing the E6 and E7 human papillomavirus oncogenes. J Control Release. 2016;231:29-37.

50. D'Abramo CM, Archambault J. Small molecule inhibitors of human papillomavirus protein-protein interactions. Open Virol J. 2011;5:80-95.

51. Zhao CY, Szekely L, Bao W, Selivanova G. Rescue of p53 function by small-molecule RITA in cervical carcinoma by blocking e6-mediated degradation. Cancer Res. 2010;70:3372-81.

52. Hengstermann A, Linares LK, Ciechanover A, Whitaker NJ, Scheffner M. Complete switch from Mdm2 to human papillomavirus E6-mediated degradation of p53 in cervical cancer cells. Proc Natl Acad Sci USA. 2001;98:1218-23.

53. Ruddock-D'Cruz NT, Prashadkumar S, Wilson KJ, Heffernan C, Cooney MA, French AJ, et al. Dynamic changes in localization of Chromobox (Cbx) family members during the maternal to embryonic transition. Mol Reprod Dev. 2008;75:477-88.

54. Charó NL, Galigniana NM, Piwien-Pilipuk G. Heterochromatin protein (HP) $1 \gamma$ is not only in the nucleus but also in the cytoplasm interacting with actin in both cell compartments. Biochim Biophys Acta Mol Cell Res. 2018;1865:432-43.

55. Le Roux LG, Moroianu J. Nuclear entry of high-risk human papillomavirus type 16 E6 oncoprotein occurs via several pathways. J Virol. 2003;77:2330-7.

56. van der Watt PJ, Maske CP, Hendricks DT, Parker MI, Denny L, Govender D, et al. The Karyopherin proteins, Crm1 and Karyopherin beta1, are overexpressed in cervical cancer and are critical for cancer cell survival and proliferation. Int $\mathrm{J}$ Cancer. 2009;124:1829-40. 